\title{
PROPERTIES OF CENTER MANIFOLDS
}

\section{BY}

\author{
JAN SIJBRAND
}

\begin{abstract}
The center manifold has a number of puzzling properties associated with the basic questions of existence, uniqueness, differentiability and analyticity which may cloud its profitable application in e.g. bifurcation theory. This paper aims to deal with some of these subtle properties.

Regarding existence and uniqueness, it is shown that the cut-off function appearing in the usual existence proof is responsible for the selection of a single center manifold, thereby hiding the inherent nonuniqueness. Conditions are given for different center manifolds at an equilibrium point to have a nonempty intersection. This intersection will include at least the families of stationary and periodic solutions crossing through the equilibrium. In the case of nonuniqueness the difference between any two center manifolds will be less than $c_{1} \exp \left(c_{2} x^{-1}\right)$ with $c_{1}$ and $c_{2}$ constants, which explains why the formal Taylor expansions of different center manifolds are the same, while the expansions do not converge.

The differentiability of a center manifold will in certain cases decrease when moving out of the origin and a simple example shows how the differentiability may be lost.

Center manifolds are usually not analytic; however, an analytic manifold may exist which contains all periodic solutions of a certain type but which may otherwise not be invariant. Using this manifold, a new and very simple proof of the Lyapunov subcenter theorem is given.
\end{abstract}

1. Introduction. The subject of bifurcation theory is the analysis of families of nontrivial solutions which branch off the trivial solution of

$$
d u / d t=L_{\lambda} u+N_{\lambda}(u) \text {. }
$$

Here $u$ is an element of some Banach space $H, L_{\lambda}$ is a linear operator which depends on $\lambda$ and $N_{\lambda}$ is a nonlinear function which is at least quadratic in $u$ for $u$ close to zero.

A family of stationary solutions may bifurcate off the family of trivial solutions $\left(u_{\lambda} \equiv 0\right)$ of (1.1) at those values of the parameter $\lambda_{0}$ for which $L_{\lambda_{0}}$ has no bounded inverse. In the case that $L_{\lambda_{0}}$ has a finite-dimensional kernel, the Lyapunov-Schmidt method reduces (1.1) to a set of equations in a finite number of variables which can be further analyzed with the Newton polygon method [49]. A typical example is the so-called pitchfork bifurcation. Applications can be found in hydrodynamics (the Taylor and Benard problems), in elasticity theory (buckling rods, plates etc.), in chemical reactor theory and morphogenesis (spatially inhomogeneous concentration distributions) and other disciplines.

Received by the editors August 23, 1982 and, in revised form, March 30, 1984.

1980 Mathematics Subject Classification. Primary 34CXX, 34DXX, 58FXX: Secondary 35BXX.

1985 American Mathematical Society $0002-9947 / 85 \$ 1.00+\$ .25$ per page 
A family of periodic solutions may bifurcate off the family of trivial solutions at parameter values $\lambda_{0}$ for which $L_{\lambda_{0}}$ has a pair of simple eigenvalues $\pm i$. This can also be formulated as: the operator $d / d t-L_{\lambda_{0}}$ has no bounded inverse in the set of $2 \pi$-periodic functions from $\mathbf{R}$ to $H$. The Lyapunov-Schmidt method can be carried out in this function space $[\mathbf{3 1}, \mathbf{4 1}]$ and typically yields a so-called Hopf bifurcation. Applications of branching families of periodic solutions appear in hydrodynamics (wavy vortices in the Taylor problem), in elasticity (flutter), in chemical and biological reactor dynamics, etc.

In a number of recent applications of bifurcation theory the two methods which have been described above do not give a complete description of the solutions of (1.1) close to the bifurcation point. In many of these problems the necessary reduction of the nonlinear problem (1.1) in the Banach space $H$ to a problem in a finite number of dimensions may be carried out following a new approach using center manifolds. In this introduction we first define the center manifold and then discuss some of its applications. Thereafter we shall outline the properties of the center manifold which will be studied in this paper.

DeFINITION 1.1. Consider the differential equation

$$
d v / d t=L v+N(v)
$$

on the Banach space $B$. Let $B=B_{1} \oplus B_{2}, \operatorname{dim} B_{1}<\infty, L\left(B_{i}\right) \subset B_{i}(i=1,2)$ and $\operatorname{Re}\left(\operatorname{spec}\left(L_{1}\right)\right)=0,0 \notin \operatorname{Re}\left(\operatorname{spec}\left(L_{2}\right)\right)$. Then a $C^{k}(k \geqslant 1)$ center manifold $S$ is a manifold which is invariant under the flow defined by (1.2) (i.e. consists of parts of trajectories of (1.2)) and is of the form

$$
S=\{(x, \sigma(x)): x \in U\} .
$$

Here $U$ is an open neighborhood of 0 in $B_{1}$ and $\sigma$ is a $k$ times continuously differentiable function from $U$ to $B_{2}$.

The requirements on $B, L$ and $N$ and the smoothness results for $\sigma$ will be made more precise in $\S 2$.

The equation (1.1) can be brought into the form (1.2) by supplying (1.1) with the equation $d \lambda / d t=0$; we identify $v=(u, \lambda), B=H \oplus \mathbf{R}, L v=\left(L_{\lambda_{0}} u, 0\right)$ and $N(v)$ $=\left(N_{\lambda}(u)+L_{\lambda} u-L_{\lambda_{0}} u, 0\right)$. Hence, the dimensions of the kernels of $L$ and $L_{\lambda_{0}}$ differ by 1 .

The center manifold approach has in common with the Lyapunov-Schmidt technique that it reduces the dimension of the problem from $\operatorname{dim} B$ to the dimension of the center manifold, which is equal to the number of critica! eigenvalues of $L$. It has an advantage over Lyapunov-Schmidt in that the reduced problem (the flow in the center manifold) still is a dynamical system, containing vital dynamical information of the full problem, as witnessed by the applications which we now consider briefly.

One example is found in the study of two-parameter dynamic bifurcation problems, as occurring for instance in reaction-diffusion theory [45] or in panel flutter analysis $[20,4]$. In this class of problems it may happen that for certain values of the parameters the linear operator obtained from linearizing the partial differential equations at a trivial solution has an eigenvalue on the imaginary axis. Usually this 
is a simple eigenvalue zero or a complex conjugate pair of imaginary eigenvalues so that the Lyapunov-Schmidt theory suffices. There is a "doubly critical" parameter pair, however, for which the critical eigenvalue zero has multiplicity two. The partial differential equation is then reduced to a pair of ordinary differential equations in the center manifold. There are parameter values close to the doubly critical parameter point for which the trivial solution of this pair of ordinary differential equations is a saddle point whose unstable and stable separatrix form a single ("homoclinic") orbit. For nearby values of the parameters a periodic solution of very large period branches off this closed orbit. For a complete description of this flow in the center manifold see $[\mathbf{2}, \mathbf{4}, \mathbf{4 5}, \mathbf{4 8}]$; at this moment it suffices to remark that the standard Lyapunov-Schmidt method is not capable of describing such behaviour. For related work see [27].

Another example (historically the first) of the reduction of a partial differential equation to an ordinary dynamical system in a finite-dimensional manifold is provided by the analysis of the successive bifurcations of solutions of the NavierStokes equations. A qualitative discussion was first given in [40]; much later the analytical details of the reduction have been provided (see [22] and the references therein). Recently, some effort has been put into the explicit computation of the center manifold in this case [39].

Although the interaction between stationary and periodic solutions and the occurrence of homoclinic orbits are the main reasons for introducing the concept of center manifolds, another reason is the geometric clarity of the idea. Several authors have been attracted by this geometric aspect of the center manifold and have treated a partial differential equation which exhibits regular Hopf bifurcation by first reducing it to an ordinary differential equation in the center manifold. As examples of this approach we mention the investigation of periodic solutions in the HodgkinHuxley model [15] and the periodic motion of a tube carrying a fluid [43]. Also, the approach of the Hopf bifurcation in $[5,30]$ is along this line.

For other discussions and applications of the center manifold see e.g. $[4,12,18,19,24,25,36]$.

The center manifold can be used successfully in a variety of applications, like those described above, but some of its properties, particularly the (non)uniqueness, limited differentiability and (non)analyticity, are subtle and not yet fully understood. This paper addresses questions regarding these properties, in particular:

(a) Existence. The question of existence has been settled by a number of authors, e.g. $[4,8,11,17,18,19,22,25,28,33]$, and this paper merely states (in $\S 2$ ) the basic existence theorem. By means of preparation for that theorem, $\S 2$ also contains a number of transformations and modifications of the original equation (1.2), leading to an integral equation for the center manifold ((2.24), (2.25)). That equation will serve as a starting point for $\$ \S 3,4$ and $5 . \S 2$ concludes by an example of a very simple bifurcation problem with a typical center manifold.

(b) Uniqueness. A center manifold may or may not be unique. Theorem 3.2 gives sufficient conditions for a center manifold to be (locally) unique. Corollary 3.3 implies that all stationary points and periodic solutions belong to the intersection of 
all center manifolds. Some further remarks on the equivalence of flows on different center manifolds, and on the unique determination of the full flow by the flow on the center manifold, are given at the end of $\S 3$.

(c) Tangency. Definition 1.1 guarantees that when there is more than one center manifold, they will all be tangent. Theorem 4.2 contains a much stronger result, stating that the difference of two center manifolds is of the order $\exp \left(-c x^{-1}\right)$ provided the algebraic and the geometric multiplicity of the critical eigenvalues of the linearized operator are equal. The difference is of the order $\exp \left(-c x^{-(2 p+1)^{-1}}\right)$ in the case that the size of the largest Jordan block related to a critical eigenvalue is $p+1$. Here $c$ is some positive constant. This transcendental tangency explains why the Taylor expansions of all center manifolds are the same.

(d) Differentiability. The example in $\$ 2$ shows that even an analytic vector field may not have a $C^{\infty}$ center manifold. An earlier example was given in [46]. However, by imposing conditions either on the vector field in a full neighborhood of the singular point, or on the flow in the center manifold, the existence of a $C^{\infty}$ center manifold may be obtained. Theorems 5.1, 5.2 and 5.4 are of this type.

(e) Analyticity. Center manifolds are rarely analytical. This is illustrated by the example in $\S 2$, and is highlighted by the expression $\exp \left(-c x^{-1}\right)$ measuring the distance between two center manifolds derived in $\S 4$. Analytic manifolds do play a role, however, in the study of periodic solutions in the neighborhood of a singular point, and this role is investigated in $\$ \S 6$ and 7 . In particular, Theorem 6.3 gives conditions to guarantee the existence of an analytic manifold containing all the families of periodic solutions with period approaching a certain limit; that manifold may otherwise not be invariant. To set the scene for Theorem 6.3, Theorems 6.1 and 6.2 contain some new results regarding the limiting behaviour of families of periodic solutions whose periods either approach a limit or else go to infinity in a wellcontrolled manner (possibly by period doubling). $\$ 7$ deals with "subcenter manifolds", which are of importance when there is more than one pair of complex conjugate imaginary eigenvalues. An important classical theorem by Lyapunov gives conditions, mainly concerning the existence of an integral of motion, which guarantee the existence of an analytic one-parameter family of periodic solutions associated with each such pair of eigenvalues. Theorem 7.1 is essentially Lyapunov's theorem, now with a very short proof showing that in this case the two-dimensional analytic manifold introduced in Theorem 6.3 is invariant and filled by periodic solutions. $\$ 7$ further analyzes the subcenter manifolds and concludes by a number of examples stressing the properties which make them stand apart from center manifolds.

Finally, $\S 8$ consists of a collection of technical proofs of various results from the preceding sections.

In order to illustrate the properties of center manifolds with model examples which are as simple as possible, all such examples in this paper will be chosen from the domain of ordinary differential equations. The results in $\$ \S 2-5$ are stated for differential equations in general Banach spaces; on the other hand, there are applications of center manifolds which do not fit into the framework presented here and which require an additional effort to assure some convergence properties (see 
the remark following equation $(2.9)$ and $[18,45])$. The subject of $\S \S 6$ and 7 seems to be relevant mainly for finite-dimensional applications, so we have restricted the statements in these sections to the finite-dimensional case.

2. Existence of the center manifold and an example of typical center manifold behaviour. In this section we formulate more explicitly the assumptions concerning the differential equation and we write down a number of modifications of this equation which put it into the form (2.24), (2.25), which is suitable for proving the existence of a center manifold. Most details of the existence proof itself are omitted as they can be found in numerous places in the literature $[4,8,11,17,18,19,22,25$, 28, 33]. The modifications as well as the formulas $(2.24),(2.25)$ will be needed in later sections for reference. This section concludes with an example of a center manifold for a simple bifurcation problem, exhibiting the rather typical behaviour of center manifolds to be discussed in $\$ \S 3-5$.

We consider a differential equation in the Banach space $B$ :

$$
d u / d t=L u+N(u) .
$$

Here $L$ is a linear operator on $B$ and $N$ is at least quadratic, i.e. there is a constant $K$ such that for $\|u\|$ small enough,

$$
\|N(u)\| \leqslant K\|u\|^{2} .
$$

In definition (1.1) we decomposed $B$ into $L$-invariant subspaces such that the restriction of $L$ to $B_{2}$ had no eigenvalues with real part zero. In this section we want to distinguish further between the subspaces associated with the eigenvalues with positive and negative real parts. So we suppose that $B$ can be decomposed into three Banach spaces

$$
B=B_{1} \oplus B_{2} \oplus B_{3}
$$

such that

$$
L\left(B_{i}\right) \subset B_{i}
$$

Define

$$
L_{i}=\left.L\right|_{B_{i}} \quad(i=1,2,3) .
$$

We are able to split (2.1) into

$$
\begin{array}{ll}
d u_{1} / d t=L_{1} u_{1}+n_{1}\left(u_{1}, u_{2}, u_{3}\right) & \left(u_{1} \in B_{1}\right), \\
d u_{2} / d t=L_{2} u_{2}+n_{2}\left(u_{1}, u_{2}, u_{3}\right) & \left(u_{2} \in B_{2}\right), \\
d u_{3} / d t=L_{3} u_{3}+n_{3}\left(u_{1}, u_{2}, u_{3}\right) & \left(u_{3} \in B_{3}\right) .
\end{array}
$$

We make the following assumptions on $B_{i}, L_{i}$ and $n_{i}$ :

$\left(\mathrm{A}_{\mathrm{i}}\right) \operatorname{dim} B_{1}<\infty$.

REMARK. This implies that for given $u_{2}$ and $u_{3}$ the equation (2.6a) represents an ordinary differential equation. 
$\left(\mathrm{A}_{\mathrm{ii}}\right)$ There is a real number $\delta>0$ such that

$$
\begin{gathered}
\operatorname{Re}\left(\operatorname{spec}\left(L_{1}\right)\right)=0, \\
\sup \operatorname{Re}\left(\operatorname{spec}\left(L_{2}\right)\right)<-\delta, \\
\text { inf } \operatorname{Re}\left(\operatorname{spec}\left(L_{3}\right)\right)>\delta .
\end{gathered}
$$

$\left(\mathrm{A}_{\mathrm{iii}}\right) L_{2}$ generates a semigroup $e^{L_{2} t}$ on $B_{2}$ which is differentiable for $t>0 ; L_{3}$ generates a semigroup $e^{L_{3} t}$ on $B_{3}$ which is differentiable for $t<0$; moreover, there is a constant $c>0$ such that

$$
\begin{array}{cc}
\left\|e^{L_{2} t}\right\|_{\mathscr{L}\left(B_{2} \rightarrow B_{2}\right)}<c e^{-\delta t} & (t>0), \\
\left\|e^{L_{3} t}\right\|_{\mathscr{L}\left(B_{3} \rightarrow B_{3}\right)}<c e^{\delta t} & (t<0) .
\end{array}
$$

(A $\left.\mathrm{A}_{\text {iv }}\right)$ The functions $n_{i}$ map $B$ into $B_{i}$ and

$$
\left\|n_{i}\left(u_{1}, u_{2}, u_{3}\right)\right\|_{B_{i}} \leqslant K\left\|\left(u_{1}, u_{2}, u_{3}\right)\right\|_{B}^{2} \text {. }
$$

RemarK. For some applications (the Navier-Stokes equations, for instance) the assumptions (2.4), (2.8a,b) and (2.9) are too restrictive. These applications have to be dealt with by introducing intermediate spaces $B_{2}^{\alpha}(0<\alpha<1)$ such that

$$
\begin{gathered}
B_{2} \subset B_{2}^{\alpha} \subset L\left(B_{2}\right), \\
\left\|e^{L_{2} t}\right\|_{\mathscr{L}\left(L\left(B_{2}\right) \rightarrow B_{2}^{\alpha}\right)}<c e^{-\delta t} / t^{\alpha}
\end{gathered}
$$

and

$$
\left\|n_{2}\left(u_{1}, u_{2}, u_{3}\right)\right\|_{L\left(B_{2}\right)}<K\left\|\left(u_{1}, u_{2}, u_{3}\right)\right\|_{B_{2}^{\alpha}}^{2} .
$$

For an extensive treatment of the center manifold problem under these circumstances, see [18].

Let us now suppose that system (2.6) possesses a $C^{k}$ center manifold at the point $u=0$. Following Definition 1.1 this manifold is a set of the form

$$
\left\{\left(u_{1}, s_{2}\left(u_{1}\right), s_{3}\left(u_{1}\right)\right): u_{1} \in U\right\},
$$

where $U$ is an open neighborhood of 0 in $B_{1}$ and where $s_{i}$ is a $C^{k}$ function from $U$ to $B_{i}(i=2,3)$. We shall derive a formula for $s_{i}(x)$.

Let $u_{1}\left(t_{0}\right) \in U$ and suppose that the $u_{1}$-component of the solution of (2.6) remains inside $U$ for $t_{0} \leqslant \tau \leqslant t$. Using the invariance property of the center manifold, we have

$$
\begin{aligned}
s_{2}\left(u_{1}(t)\right)= & u_{2}(t)=e^{L_{2}\left(t-t_{0}\right)} s_{2}\left(u_{1}\left(t_{0}\right)\right) \\
& +\int_{t_{0}}^{t} e^{L_{2}(t-\tau)} n_{2}\left(u_{1}(\tau), s_{2}\left(u_{1}(\tau)\right), s_{3}\left(u_{1}(\tau)\right)\right) d \tau .
\end{aligned}
$$

By choosing $t=0$ and taking the limit for $t_{0} \rightarrow-\infty$ we obtain

$$
s_{2}\left(u_{1}(0)\right)=\int_{-\infty}^{0} e^{-L_{2} \tau} n_{2}\left(u_{1}(\tau), s_{2}\left(u_{1}(\tau)\right), s_{3}\left(u_{1}(\tau)\right)\right) d \tau
$$

Similarly, for $s_{3}$ we obtain

$$
s_{3}(x(0))=-\int_{0}^{\infty} e^{-L_{3} \tau} n_{3}\left(u_{1}(\tau), s_{2}\left(u_{1}(\tau)\right), s_{3}\left(u_{1}(\tau)\right)\right) d \tau
$$


while $u_{1}(t)$ has to satisfy for $-\infty<t<\infty$,

$$
d u_{1} / d t=L_{1} u_{1}+n_{1}\left(u_{1}, s_{2}\left(u_{1}\right), s_{3}\left(u_{1}\right)\right) .
$$

This kind of formulas for $s_{2}, s_{3}$ was first written down in [6 and 35].

Taking the limit $t_{0} \rightarrow \pm \infty$ makes sense only if (i) the solution of (2.17) exists on the entire $t$-axis, (ii) the integrals in (2.15) and (2.16) converge, (iii) the quantity $s_{2}\left(u_{1}\left(t_{0}\right)\right)$ remains bounded when $t_{0} \rightarrow \pm \infty$. Because these requirements are not met by arbitrary nonlinearities $n_{i}(i=1,2,3)$ we modify the differential equations (2.6) as follows (cf. [14, p. 612]).

Let $\chi:[0, \infty) \rightarrow \mathbf{R}$ be a $C^{\infty}$ function with the additional properties

$$
\begin{aligned}
& 0 \leqslant \chi(v) \leqslant 1 \quad \text { for all } v \geqslant 0, \\
& \chi(v)=1 \text { for all } v \in[0,1], \\
& \chi(v)=0 \text { for all } v \in[2, \infty)
\end{aligned}
$$

Let $\varepsilon$ be an arbitrary positive parameter. Consider the equations

$$
\begin{aligned}
& d u_{1} / d t=L_{1} u_{1}+n_{1}\left(u_{1}, u_{2}, u_{3}\right) \chi\left(\left\|u_{1}\right\| / \varepsilon\right), \\
& d u_{2} / d t=L_{2} u_{2}+n_{2}\left(u_{1}, u_{2}, u_{3}\right) \chi\left(\left\|u_{1}\right\| / \varepsilon\right), \\
& d u_{3} / d t=L_{3} u_{3}+n_{3}\left(u_{1}, u_{2}, u_{3}\right) \chi\left(\left\|u_{1}\right\| / \varepsilon\right) .
\end{aligned}
$$

If we now repeat the procedure indicated by the equations $(2.14)-(2.17)$ for the system (2.19) rather than (2.6), we find that the requirements (i) and (ii) are fulfilled. If we search for a global center manifold $S_{\varepsilon}$ of (2.19), i.e.

$$
S_{\varepsilon}=\left\{\left(u_{1}, s_{2, \varepsilon}\left(u_{1}\right), s_{3, \varepsilon}\left(u_{1}\right)\right): u_{1} \in B_{1}\right\} \text {, }
$$

where $s_{2, \varepsilon}$ and $s_{3, \varepsilon}$ are defined and bounded on all of $B_{1}$, then requirement (iii) will be met as well.

If $S_{\varepsilon}$ is a center manifold of (2.19) of the kind (2.20), then the restriction of $S_{\varepsilon}$ to $U \cap D_{\varepsilon}$ (where $D_{\varepsilon}$ is the ball of radius $\varepsilon$ around 0 in $B_{1}$ ) is a center manifold of (2.6).

We perform a scaling

$$
\begin{gathered}
u_{1}=\varepsilon x, \quad u_{2}=\varepsilon y, \quad u_{3}=\varepsilon z, \\
s_{i, \varepsilon}\left(u_{1}\right)=\varepsilon \sigma_{i}(x ; \varepsilon)
\end{gathered}
$$

and

$$
n_{i}\left(u_{1}, u_{2}, u_{3}\right) \chi\left(\left\|u_{1}\right\| / \varepsilon\right)=\varepsilon g_{i}(x, y, z ; \varepsilon) .
$$

The modified and scaled version of (2.15), (2.16), (2.17) now becomes

$$
\begin{aligned}
& \sigma_{2}(\xi ; \varepsilon)=\int_{-\infty}^{0} e^{-L_{2} \tau} g_{2}(x(\tau ; \xi, \sigma, \varepsilon), \sigma(x(\tau ; \xi, \sigma, \varepsilon) ; \varepsilon) ; \varepsilon) d \tau, \\
& \sigma_{3}(\xi ; \varepsilon)=-\int_{0}^{\infty} e^{-L_{3} \tau} g_{3}(x(\tau ; \xi, \sigma, \varepsilon), \sigma(x(\tau ; \xi, \sigma, \varepsilon) ; \varepsilon) ; \varepsilon) d \tau,
\end{aligned}
$$

where $x(t ; \xi, \sigma, \varepsilon)$ is the solution of

$$
d x / d t=L_{1} x+g_{1}(x, \sigma(x ; \varepsilon) ; \varepsilon), \quad x(0)=\xi .
$$

In the argument of $g_{i}$ we have abbreviated the pair $\sigma_{2}, \sigma_{3}$ by $\sigma$.

We summarize the analysis of this section up to this point: if $S_{\varepsilon}$ is a global center manifold of (2.19) and the functions $s_{2, \varepsilon}$ and $s_{3, \varepsilon}$ in (2.20) are bounded on $B_{1}$, then the corresponding scaled functions $\sigma_{2}(\xi, \varepsilon)$ and $\sigma_{3}(\xi ; \varepsilon)$ satisfy $(2.24),(2.25)$. 
Conversely, if (2.24), (2.25) possesses a bounded solution $\sigma_{2}(\xi ; \varepsilon), \sigma_{3}(\xi ; \varepsilon)$ defined for all $\xi \in B_{1}$, then the functions $s_{i, \varepsilon}\left(u_{1}\right)$ from (2.22) define a center manifold of (2.6) for $u_{1} \in U \cap D_{\varepsilon}$. This can be concluded from the autonomous character of $(2.25)$ :

$$
x(t+\tau ; \xi, \sigma, \varepsilon)=x(\tau ; x(t ; \xi, \sigma, \varepsilon), \sigma, \varepsilon)
$$

whence

$$
\sigma(x(t ; \xi, \sigma, \varepsilon) ; \varepsilon)=\int_{-\infty}^{t} e^{-L_{2}(s-t)} g_{2}(x(s ; \xi, \sigma, \varepsilon), \sigma(x(s ; \xi, \sigma, \varepsilon)) ; \varepsilon) d s .
$$

The right-hand side of (2.27) is differentiable with respect to $t$ and its derivative is

$$
L_{2} \sigma(x ; \varepsilon)+g_{2}(x, \sigma(x ; \varepsilon) ; \varepsilon),
$$

which implies that the solution of (2.24), (2.25) defines an invariant manifold indeed. The tangency property follows easily from (2.9) and (2.23).

We now define some function spaces which are useful in the search for the solution of (2.24), (2.25). Finally, we state the main theorem of this section which establishes the existence and regularity of a solution of (2.24), (2.25).

Definitions. (i) $\Sigma^{(k)}$ is the space of $k$ times continuously differentiable functions from $B_{1}$ to $B_{2}+B_{3}$ with norm

$$
\|\boldsymbol{\sigma}\|_{k}=\sup \left\{\sum_{i=0}^{k}\left\|\frac{d^{P} \sigma}{d x^{p}}(x)\right\|: x \in B_{1}\right\} .
$$

$$
\Sigma_{R}^{(k)}=\left\{\sigma \in \Sigma^{(k)}:\|\sigma\|_{k}<R\right\}
$$

(iii)

$$
\begin{aligned}
\sum_{R, l}^{(k)}=\left\{\sigma \in \Sigma_{R}^{(k)}: \forall x_{1}, x_{2} \in B_{1}:\right. \\
\\
\left.\left\|d^{k} \sigma\left(x_{1}\right) / d x^{k}-d^{k} \sigma\left(x_{2}\right) / d x^{k}\right\| \leqslant l\left\|x_{1}-x_{2}\right\|\right\} .
\end{aligned}
$$

THEOREM 2.1. Let $B_{i}, L_{i}$ and $n_{i}$ be defined in (2.3) and (2.4) and let $n_{i}$ have derivatives of order up to $k(k \geqslant 1)$. Let assumptions $\left(\mathrm{A}_{\mathrm{i}-\mathrm{iv}}\right)$ be satisfied. Suppose that there are real functions $r_{i}(\delta)$ such that, for all $v, w \in B$ with $\|v\|<\delta$ and $\|w\|<\delta$,

$$
\left\|d^{k} n_{i}(v) / d u^{k}-d^{k} n_{i}(w) / d u^{k}\right\| \leqslant r_{i}(\delta)\|v-w\| \quad(i=1,2,3) .
$$

Moreover, assume that there are functions $\rho_{i}(\delta)$ which tend to 0 when $\delta$ approaches 0 and for which

$$
\left\|n_{i}(v)-n_{i}(w)\right\| \leqslant \rho_{i}(\delta)\|v-w\| .
$$

Then there are positive numbers $\varepsilon_{0}, R, l$ such that for $\varepsilon \in\left(0, \varepsilon_{0}\right)$ the set of equations (2.24), (2.25) has a unique solution $\sigma \in \Sigma_{R, l}^{(k)}$.

Proof. The proof concerns the operator $T_{\varepsilon}$ defined by

$$
\begin{aligned}
\left(T_{\varepsilon} \sigma\right)(\xi)=\left(\int_{-\infty}^{0} e^{-L_{2} \tau} g_{2}(x(\tau ; \xi, \sigma, \varepsilon), \sigma(x(\tau ; \xi, \sigma, \varepsilon)) ; \varepsilon) d \tau\right. \\
\left.-\int_{0}^{\infty} e^{-L_{3} \tau} g_{3}(x(\tau ; \xi, \sigma, \varepsilon), \sigma(x(\tau ; \xi, \sigma, \varepsilon)) ; \varepsilon) d \tau\right)
\end{aligned}
$$


where $x(t ; \xi, \sigma, \varepsilon)$ is the solution of (2.25). Using Kelley's techniques [25, proofs of Lemmas 4 and 5] (see also [33]), which are easily adapted to the use of Lipschitz spaces, one is able to prove that:

1. For every $l>0$ we can choose $\varepsilon_{0}$ and $R$ so small that, for all $\varepsilon$ with $0<\varepsilon<\varepsilon_{0}$,

$$
T_{\varepsilon} \Sigma_{R, l}^{(0)} \subset \Sigma_{R, l}^{(0)} \text {. }
$$

2. For every $l>0$ we can choose $\varepsilon_{0}$ and $R$ so small that (2.35) is satisfied and $T_{\varepsilon}$ is a contraction in $\Sigma_{R, l}^{(0)}$.

3. For every $l>0$ we can choose $\varepsilon_{0}$ and $R$ so small that there are numbers $R_{1}, \ldots, R_{k}$ such that for all $\varepsilon, 0<\varepsilon<\varepsilon_{0}, T_{\varepsilon}$ maps the set

$$
\Sigma_{R, l}^{(0)} \cap \Sigma_{R_{1}, l}^{(1)} \cap \cdots \cdots \cap \Sigma_{R_{k}, l}^{(k)}
$$

into itself and $T_{\varepsilon}$ is a contraction on this set.

Point 2 proves the existence of a unique center manifold of (2.24), (2.25); 3 supplies the regularity of this manifold. This completes the proof of Theorem 2.1.

We conclude this section by an example of a bifurcation problem with a center manifold which exhibits some of the properties which we want to deal with in the sequel.

Consider the set of equations

$$
\begin{gathered}
d x / d t=\lambda x-x^{2} \\
d y / d t=-y+x^{2}, \\
d \lambda / d t=0 .
\end{gathered}
$$

In this example, $B_{1}$ is the $(x, \lambda)$-plane and $B_{2}$ is the $y$-axis. For every fixed $\lambda$ we can study the phase plane of the pair of equations (2.37a), (2.37b); see Figure 1 .

For $\lambda_{0}<0$ the trivial solution is a node and the solution $(x, y)=\left(\lambda_{0}, \lambda_{0}^{2}\right)$ is a saddle point. For $\lambda_{0} \in(-1,0)$ all trajectories which approach the node for $t \rightarrow \infty$ are tangent to the line $\lambda=\lambda_{0}, y=0, x \in \mathbf{R}$.

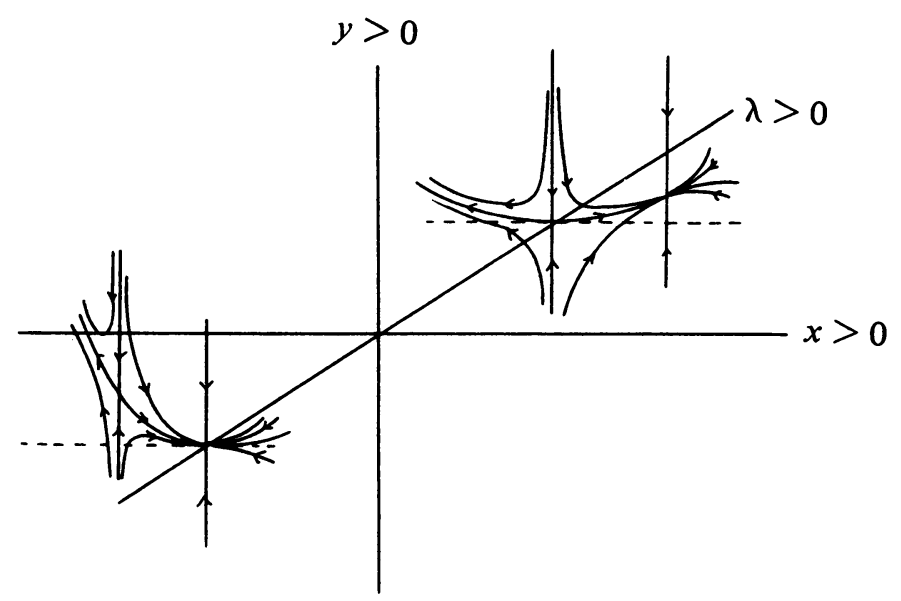

FIGURE 1 
For $\lambda_{0}>0$ the trivial solution is a saddle and the solution $(x, y)=\left(\lambda_{0}, \lambda_{0}^{2}\right)$ is a node. There has been an "exchange of stabilities" at the bifurcation point $\lambda=0$. For $\lambda_{0} \in(0,1)$ all trajectories which approach the node for $t \rightarrow \infty$ are in the plane $\lambda=\lambda_{0}$ and tangent to the line through the node with slope $2 \lambda_{0}\left(1-\lambda_{0}\right)^{-1}$.

A center manifold of (2.37) will be a manifold of the form

$$
S=\{(x, \lambda, \sigma(x, \lambda)):(x, \lambda) \in U\},
$$

where $U$ is a neighborhood of 0 in the $(x, \lambda)$-plane and $\sigma$ is a mapping from $U$ to the $y$-axis.

On the quadrant $x<0, \lambda<0$ the function $\sigma$ is uniquely determined; here $S$ coincides with the unstable saddle separatrices. On the quadrant $x>0, \lambda<0$ we are free to take any of the trajectories which approach the nodes for $t \rightarrow+\infty$. On the domain $\lambda>0, x<\lambda, S$ is unique and, for $\lambda>0, x>\lambda$, it is not.

We conclude: in every neighborhood $U$ of 0 there are subneighborhoods where $S$ is unique and others where $S$ is not unique. We call this property local (non)uniqueness of center manifolds.

We now study the differentiability of $\sigma$. From (2.37a), (2.37b) we get

$$
\left(\lambda x-x^{2}\right)(d \sigma / d x)=-\sigma+x^{2} .
$$

The solution of this singular equation near $x=0$ can be written as

$$
\sigma(x, \lambda)=\sum_{n=2}^{\infty} a_{n}(\lambda) x^{n}+c\left|\frac{\lambda-x}{x}\right|^{1 / \lambda},
$$

provided $\lambda^{-1}$ is not an integer.

Now let $\lambda<0$. The coefficient $c$ is arbitrary, which represents the fact that many trajectories approach 0 . For one value of $c$ (say $c_{s}(\lambda)$ ), (2.40) represents the saddle separatrix which connects the two singular points.

The coefficients $a_{n}(\lambda)$ are determined from the recurrence relation $(n \lambda+1) a_{n}=$ $a_{n-1}(n \geqslant 3)$ and from $a_{2}=(2 \lambda+1)^{-1}$. The radius of convergence of the series is $|\lambda|$ and for $x=\lambda$ the series diverges. This implies that

$$
c_{s}(\lambda) \neq 0 \text {. }
$$

Another way to reach this result is to write down the variation of constants formula for the solution of (2.39); after some partial integration one obtains

$$
c_{s}(\lambda)=(-1)^{n} \frac{1+\lambda}{1+n \lambda} \cdot \lambda \int_{0}^{1} \eta^{n+1 / \lambda}(1-\eta)^{-n-1 / \lambda} d \eta
$$

where $n$ is such that $-1<n+1 / \lambda<0$.

From (2.41) we conclude that for given $\lambda_{0}<0, \lambda_{0}^{-1}$ not an integer, the function $\sigma\left(x, \lambda_{0}\right)$ consists of an analytic part plus a term which behaves like $x^{-1 / \lambda_{0}}$ near $x=0$. This means that $\sigma\left(x, \lambda_{0}\right)$ is no more than entier $\left(-1 / \lambda_{0}\right)$ times continuously differentiable in the point $(x, \lambda)=\left(0, \lambda_{0}\right)$. So along the line of nodes there is a singularity in an ever higher derivative of $\sigma$ when we approach 0 : we conclude that there does not exist a $C^{\infty}$ center manifold of (2.37), and a fortiori no analytic center manifold.

If $\lambda^{-1}$ is an integer, then the term $c\left|(\lambda-x) x^{-1}\right|^{\lambda^{-1}}$ does no harm at $x=0$, but then the power series contains a term of the order $x^{N} \log x, N=-\lambda^{-1}$. 
3. Uniqueness, nonuniqueness and local uniqueness of center manifolds. In this section we study dynamical systems with many center manifolds and the influence of the modification of a dynamical system outside a neighborhood of zero on the center manifolds of that system.

We first give some examples showing that this modification leads to a unique (global) center manifold of the modified system. Then we show (Theorem 3.1) that for every single center manifold there is a modification of the dynamical system making that center manifold the unique global one. In Theorem 3.2 we conclude that solutions which stay in a neighborhood of zero in a center manifold do not feel this modification and are therefore contained in every center manifold. This is one of the properties which makes the center manifold useful for applications.

Anosov (see [25]) gives the following simple dynamical system:

$$
d x / d t=x^{2} ; \quad d y / d t=-y .
$$

The trajectories in the phase plane are the curves $y=\alpha e^{1 / x}$, where $\alpha$ is an arbitrary real parameter. If $U$ is an open neighborhood of 0 in $\mathbf{R}$, then every manifold $S_{\alpha}$ is a $C^{\infty}$ cenier manifold of (3.1), where for $\alpha \in \mathbf{R}$,

$$
S_{\alpha}=\left\{\left(x, \alpha e^{1 / x}\right): x \in U, x<0\right\} \cup\{(x, 0): x \in U, x \geqslant 0\} .
$$

This example clearly shows that center manifolds are not always unique; the more complicated dynamical system (2.37) is another example of this phenomenon.

Next, we study the dynamical system

$$
d x / d t=x^{2} ; \quad d y / d t=-y+x^{2} .
$$

This system has the same topological properties as (3.1) but it serves better to illustrate the effect of a cut-off function. Let $\chi:[0, \infty) \rightarrow \mathbf{R}$ be a $C^{\infty}$ function as defined in (2.15), i.e.

$$
\begin{aligned}
& 0 \leqslant \chi(v) \leqslant 1 \text { for all } v \geqslant 0 \\
& \chi(v)=1 \text { for all } v \in[0,1], \\
& \chi(v)=0 \text { for all } v \in[2, \infty)
\end{aligned}
$$
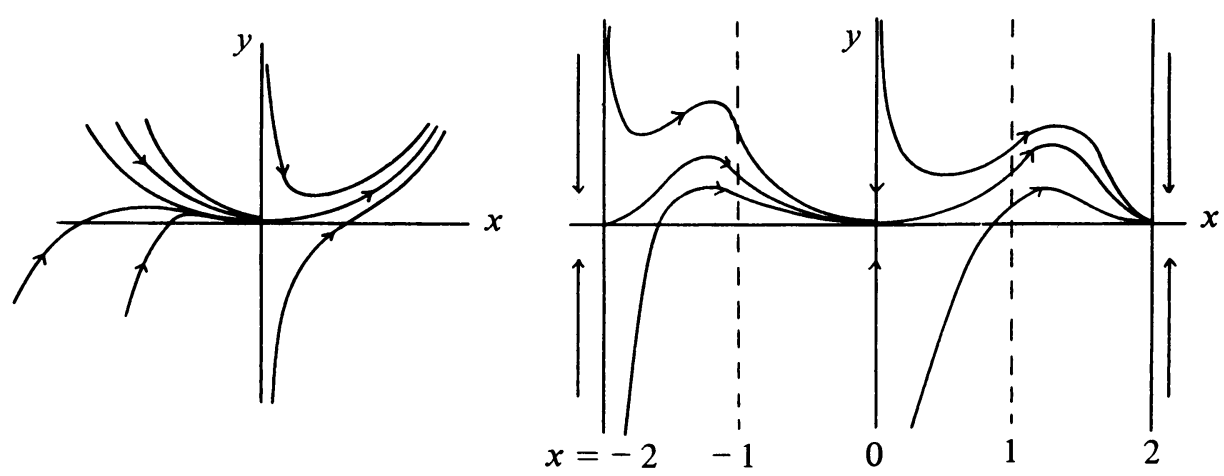

Figure 2 

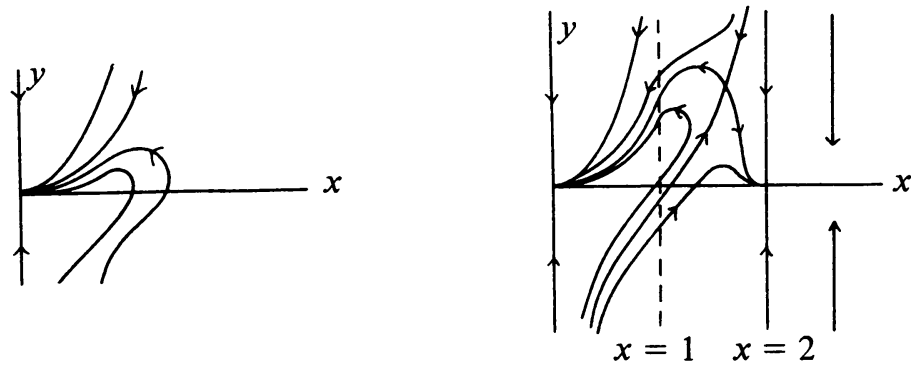

FIGURE 3

The trajectories of (3.3) as well as those of the modified system

$$
d x / d t=x^{2} \chi(|x|) ; \quad d y / d t=-y+x^{2} \chi(|x|) .
$$

appear in Figure 2.

Clearly, there is a unique global center manifold (i.e. defined for all $x$, compare (2.20)) of the modified system. The cut-off function has forced this uniqueness upon the system (3.4) and it has enabled us to prove existence of a center manifold using a contraction argument in the proof of Theorem 2.1.

A slightly more complicated situation arises in the following example (which we treat here for later reference):

$$
d x / d t=x^{3}-y x ; \quad d y / d t=-y+2 x^{2} .
$$

In Figure 3 we display the right-hand side of the phase portrait of (3.5) (the left-hand side is its mirror image) as well as the corresponding picture of

$$
d x / d t=\left(x^{3}-y x\right) \chi(|x|) ; \quad d y / d t=-y+2 x^{2} \chi(|x|) .
$$

Again, the cut-off function has forced the uniqueness of a global center manifold. The modified system has the additional feature of a stationary point which bears no relation with the first zero (at $x=2$ ) of the cut-off function.

It will be clear from the above examples that a different function $\chi$ will generally give rise to the selection of a different center manifold. The following theorem is sort of a converse of this remark; we need it for several proofs in this section and in the next one.

THEOREM 3.1. Consider the system (2.6); suppose that the assumptions $\left(\mathrm{A}_{\mathrm{i}-\mathrm{iv}}\right)$ in $\$ 2$ are satisfied and that, additionally, $n_{i} \in C^{k}(k \geqslant 1)$. Let $S$ be a given $C^{k}$ center manifold of (2.6):

$$
S=\left\{\left(u_{1}, s_{1}\left(u_{1}\right), s_{2}\left(u_{2}\right)\right): u_{1} \in U\right\} .
$$

Here $U$ is a neighborhood of 0 in $B_{1}$. Then there is a system

$$
\begin{array}{ll}
d u_{1} / d t=L_{1} u_{1}+\tilde{n}_{1}\left(u_{1}, u_{2}, u_{3}\right) & \left(u_{1} \in B_{1}\right), \\
d u_{2} / d t=L_{2} u_{2}+\tilde{n}_{2}\left(u_{1}, u_{2}, u_{3}\right) & \left(u_{2} \in B_{2}\right), \\
d u_{3} / d t=L_{3} u_{3}+\tilde{n}_{3}\left(u_{1}, u_{2}, u_{3}\right) & \left(u_{3} \in B_{3}\right),
\end{array}
$$


and a neighborhood $\tilde{U} \subset U$ such that the $C^{k-1}$ functions $\tilde{n}_{i}$ coincide with $n_{i}$ for $u_{1} \in \tilde{U}$ and $\tilde{n}_{i}=0$ for $u_{1}$ outside $U$, while (3.7) has a unique global center manifold $\tilde{S}$. Moreover, the restrictions of $\tilde{S}$ and $S$ to $\tilde{U}$ coincide.

Proof. Let $U_{1}$ be a neighborhood of 0 in $B_{1}$ such that $U_{1} \subset U$. Let $\psi: B_{1} \rightarrow \mathbf{R}$ be a $C^{\infty}$ cut-off function such that $\psi=1$ inside $U_{1}$ and $\psi=0$ outside $U$. Introduce a global transformation of coordinates on $B$ by

$$
\begin{aligned}
& v_{1}=u_{1}, \\
& v_{2}=u_{2}-s_{2}\left(u_{1}\right) \psi\left(u_{1}\right), \\
& v_{3}=u_{3}-s_{3}\left(u_{1}\right) \psi\left(u_{1}\right) .
\end{aligned}
$$

Following [33, proof of Lemma 1] we find that the resulting differential equations for $v_{1}, v_{2}, v_{3}$ have right-hand sides in $C^{k-1}$ and possess a (not necessarily unique) local center manifold

$$
S^{*}=\left\{\left(v_{1}, 0,0\right): v_{1} \in U_{1}\right\} .
$$

Choosing now a neighborhood $U_{2}$ of 0 in $B_{1}, U_{2} \subset U_{1}$ and cutting off the equations for $v_{1}, v_{2}, v_{3}$ in the same fashion as (2.19) by another $C^{\infty}$ function $\chi$ such that $\chi=1$ inside $U_{2}$ and $\chi=0$ outside $U_{1}$, we end up with a dynamical system for $v_{1}, v_{2}, v_{3}$ with a uniquely determined globally defined center manifold

$$
\hat{S}=\left\{\left(v_{1}, 0,0\right): v_{1} \in B_{1}\right\} \text {. }
$$

Now transforming this dynamical system back to $u_{1}, u_{2}, u_{3}$ coordinates by means of (3.8) we turn $\hat{S}$ into the desired manifold $\tilde{S}$ and produce the right-hand sides $\tilde{n}_{i} \in C^{k-1}$. This completes the proof of Theorem 3.1.

We shall use Theorem 3.1 combined with the contraction mapping argument from the proof of Theorem 2.1 to give a new and simple proof of the following theorem; for a discussion of alternative proofs see the remark at the end of this section.

THEOREM 3.2. Consider the equation (2.6), where $L_{i}, B_{i}, n_{i}(i=1,2)$ satisfy conditions $\left(\mathrm{A}_{\mathrm{i}-\mathrm{iv}}\right)$ in $\$ 2$, with $n_{i} \in C^{k}(k \geqslant 1)$, and suppose that $L$ has no eigenvalues with positive real part, i.e. $B_{3}=\{0\}$ and

$$
d u_{1} / d t=L_{1} u_{1}+n_{1}\left(u_{1}, u_{2}\right), \quad d u_{2} / d t=L_{2} u_{2}+n_{2}\left(u_{1}, u_{2}\right) .
$$

Suppose that $\sigma_{A}$ and $\sigma_{B}$ are $C^{k}$ functions from a neighborhood $U$ of 0 in $B_{1}$ to $B_{2}$ such that

$$
S_{A}=\left\{\left(x, \sigma_{A}(x)\right): x \in U\right\}
$$

and

$$
S_{B}=\left\{\left(x, \sigma_{B}(x)\right): x \in U\right\}
$$

are center manifolds of (3.11). Let $u_{1}(t)$ denote a solution of

$$
d u_{1} / d t=L_{1} u_{1}+n_{1}\left(u_{1}, \sigma_{A}\left(u_{1}\right)\right) .
$$

Then there is a neighborhood $U^{\prime} \subset U$ of 0 such that

$$
\left\{u_{1}(t):-\infty<t \leqslant 0\right\} \subset U^{\prime}
$$

implies

$$
\sigma_{A}\left(u_{1}(t)\right)=\sigma_{B}\left(u_{1}(t)\right) \text { for all } t,-\infty<t \leqslant 0 \text {. }
$$


REMARK. This theorem can be readily verified for the examples (2.37), (3.3) and (3.5) where the center manifold is indeed unique precisely there where the trajectories stay close to the singular point for $t \rightarrow-\infty$.

Proof of Theorem 3.2. By Theorem 3.1 there is a modification of (3.11) and a neighborhood $U^{\prime} \subset U$ such that $\left.S_{B}\right|_{U^{\prime}}$ is a submanifold of the globally unique center manifold of

$$
d u_{1} / d t=L_{1} u_{1}+\tilde{n}_{1}\left(u_{1}, u_{2}\right), \quad d u_{2} / d t=L_{2} u_{2}+\tilde{n}_{2}\left(u_{1}, u_{2}\right) .
$$

From the contraction property of the operator $T_{\varepsilon}$ defined in the proof of Theorem 2.1 , the iteration

$$
\sigma^{(n+1)}(\xi)=\int_{-\infty}^{0} e^{-L_{2} s} \tilde{n}_{2}\left(u_{1}\left(\tau ; \sigma^{(n)}\right), \sigma^{(n)}\left(u_{1}\left(\tau ; \sigma^{(n)}\right)\right)\right) d \tau
$$

with $u_{1}(t ; \sigma)$ the solution of

$$
d u_{1} / d t=L_{1} u_{1}+\tilde{n}_{1}\left(u_{1}, \sigma^{(n)}\left(u_{1}\right)\right) ; \quad u_{1}(0)=\xi,
$$

converges to $\sigma_{B}(\xi)$ if $\left\|\sigma^{(0)}\right\|$ is small enough and $\xi \in U^{\prime}$. Now let us start this iteration with $\sigma^{(0)}=\sigma_{A}$. By the assumption (3.14) made on the solution of (3.13) we have, for $\xi=u_{1}\left(0 ; \sigma_{A}\right)$,

$$
\begin{aligned}
\sigma^{(1)}(\xi) & =\int_{-\infty}^{0} e^{-L_{2} s} n_{2}\left(u_{1}\left(\tau ; \sigma_{A}\right), \sigma_{A}\left(u_{1}\left(\tau ; \sigma_{A}\right)\right)\right) d \tau \\
& =\int_{-\infty}^{0} e^{-L_{2} s}\left(d / d \tau-L_{2}\right) \sigma_{A}\left(u_{1}\left(\tau ; \sigma_{A}\right)\right) d \tau=\sigma_{A}(\xi) .
\end{aligned}
$$

Hence, all iterands $\sigma^{(n)}(\xi)$ are equal to $\sigma_{A}(\xi)$ which implies that $\sigma_{B}(\xi)=$ $\lim _{n \rightarrow \infty} \sigma^{(n)}(\xi)=\sigma_{A}(\xi)$.

THEOREM 3.2'. (i) If the linear operator $L$ in (2.1) does not have eigenvalues with negative real part, then the statement (3.15) holds if

$$
\left\{u_{1}(t): 0 \leqslant t<\infty\right\} \subset U^{\prime} .
$$

(ii) If $L$ has eigenvalues with negative and positive real parts, then (3.15) holds if

$$
\left\{u_{1}(t):-\infty<t<\infty\right\} \subset U^{\prime} .
$$

COROLlARY 3.3. Every center manifold contains all stationary points and periodic solutions which are close enough to 0 (see also [4, 27]).

REMARK 1. An alternative proof of Theorem 3.2 can be constructed by first proving the existence of a collection of smooth fibers which are transversal to every center manifold and which are transported by the flow of (3.11), while the flow in each fiber is exponentially attracting for $t \rightarrow+\infty$ with approximately the rate prescribed by the eigenvalues of $L_{2}[4,8,12,34,36]$. For $t \rightarrow-\infty$ the flow in the fibers is expanding and for solutions which remain bounded for all negative $t$ this implies that the corresponding fibers intersect with at most one center manifold.

REMARK 2. In [34, \$4c] it is shown by means of the techniques mentioned in the previous remark that the flow on the center manifold uniquely defines the full flow of (2.6) in a neighborhood of 0 , i.e. the flow determined by (2.6) with initial values also outside the center manifold. More precisely, it is shown that two different 
dynamical systems, which have center manifolds on which the flows are "equivalent" in a certain sense, have "equivalent" flows in a full neighborhood of 0 .

REMARK 3. The same invariant foliation techniques might be useful in proving that the flows on two different center manifolds of the same dynamical system (in the case of nonuniqueness, of course) are equivalent.

REMARK 4. The formulation of Theorem 3.2 does not exclude the existence of solutions of (3.11) which are bounded for all $t<0$ but whose orbit is not contained in any center manifold. This situation will be proved to be impossible as a special consequence of the analysis in $§ 8.2$.

4. The order of tangency of two center manifolds. In this section we prove that the difference between two center manifolds $\sigma_{A}(x)$ and $\sigma_{B}(x)$ is of the order $\exp (-c /\|x\|)$ provided that the algebraic and geometric multiplicities of the critical eigenvalues are equal. In the case that these multiplicities are not equal the difference is of the order $\exp \left(-c /\|x\|^{1 /(2 p+1)}\right)$, where $p+1$ is the size of the largest Jordan block related to a critical eigenvalue. Similar estimates hold for the derivatives of center manifolds.

In both cases the difference is smaller than any order $\|x\|^{N}$; this was concluded from an entirely different analysis in [11 and 51, Theorem 9.1]. It explains why the formal Taylor expansion for all center manifolds is the same.

In this section we first give a heuristic treatment and we then formulate the precise results.

Consider the case of a dynamical system (2.6) with $B_{3}=\{0\}$ (i.e. there are no eigenvalues with positive real part). A center manifold $S$ of the form $S=\left\{\left(u_{1}, \sigma\left(u_{1}\right)\right)\right.$ : $\left.u_{1} \in B_{1}\right\}$ will satisfy

$$
\sigma(\xi)=\int_{-\infty}^{0} e^{-L_{2} \tau} n_{2}\left(u_{1}(\tau ; \xi), \sigma\left(u_{1}(\tau ; \xi)\right)\right) \chi\left(\left\|u_{1}(\tau ; \xi)\right\|\right) d \tau
$$

Here for $t \leqslant 0, u_{1}(t ; \xi)$ is the solution of

$$
d u_{1} / d t=L_{1} u_{1}+n_{1}\left(u_{1}, \sigma\left(u_{1}\right)\right) \chi\left(\left\|u_{1}\right\|\right) ; \quad u_{1}(0)=\xi .
$$

Usually different cut-off functions $\chi$, say $\chi_{A}$ and $\chi_{B}$, will lead to different solutions for $\sigma$, say $\sigma_{A}$ and $\sigma_{B}$ (compare $\S 3$ ).

We now discuss two ways to estimate the difference between $\sigma_{A}$ and $\sigma_{B}$.

The first point of view is the following. Let $t_{0}(\xi)$ be the first negative value of $t$ for which $\chi_{A}\left(\left\|u_{1}(t ; \xi)\right\|\right) \neq \chi_{B}\left(\left\|u_{1}(t ; \xi)\right\|\right)$. The integrands in (4.1) will be slightly different for $t_{0} \leqslant t \leqslant 0$ due to the fact that $\sigma$ appears explicitly, but the main difference between $\sigma_{A}$ and $\sigma_{B}$ will be caused for values of $t$ smaller than $t_{0}$, so, roughly,

$$
\sigma_{A}(\xi)-\sigma_{B}(\xi) \approx \int_{-\infty}^{t_{0}} e^{-L_{2} \tau} n_{2}\left(\chi_{A}-\chi_{B}\right) d \tau .
$$

Using (2.8a) we have $\sigma_{A}(\xi)-\sigma_{B}(\xi)=O\left(e^{\delta t_{0}}\right)$. Of course, $t_{0}$ depends on the initial value $\xi$. For example, if $(4.2)$ is as simple as $\dot{u}_{1}=-u_{1}^{2}, u_{1}(0)=\xi>0$ with solution $u_{1}(t)=\xi(1+\xi t)^{-1}$, then $t_{0}$ will be of the order $-\xi^{-1}$ and so

$$
\sigma_{A}(\xi)-\sigma_{B}(\xi)=O(\exp (- \text { const } / \xi)) \text {. }
$$


A second point of view will now be discussed. We apply the transformation $x=u_{1} ; w=u_{2}-\sigma_{A}\left(u_{1}\right)$. System (2.6) now obtains the form

$$
d x / d t=L_{1} x+h_{1}(x, w), \quad d w / d t=L_{2} w+h_{2}(x, w)
$$

where $h_{2}(x, 0) \equiv 0$. Let $x_{0} \in B_{1}$ be arbitrary and let $\left(x\left(t ; x_{0}\right), w\left(t ; x_{0}\right)\right)$ be the solution of (4.5) with $w\left(0 ; x_{0}\right)=\sigma_{B}\left(x_{0}\right)-\sigma_{A}\left(x_{0}\right)$. Let $\varepsilon$ be the radius of a neighborhood of 0 in $B_{1}$ on which the cut-off functions for $\sigma_{A}$ and $\sigma_{B}$ are equal. There are two possibilities:

Case 1. There is a $t_{1}<0$ such that $\left\|x\left(t_{1} ; x_{0}\right)\right\|=\varepsilon$. In the simple situation that $\operatorname{dim} B_{1}=1$ we have the estimate

$$
d|x| / d t \geqslant-k x^{2}
$$

which implies that, for all $t \geqslant 0$ for which $x(t ; \xi)$ exists,

$$
|x(t ; \xi)| \geqslant|\xi| /(1+k|\xi| t),
$$

hence

$$
\left|x_{0}\right|=\left|x\left(-t_{1} ; x\left(t_{1} ; x_{0}\right)\right)\right| \geqslant \varepsilon /\left(1-k \varepsilon t_{1}\right) .
$$

For the $w$-component we have the estimate

$$
\left\|w_{0}\right\|=\left\|w\left(-t_{1} ; w\left(t_{1} ; w_{0}\right)\right)\right\| \leqslant D\left\|w\left(t_{1} ; x_{0}\right)\right\| \exp (\delta-\eta) t_{1}
$$

for some $\eta \in(0, \delta)$ and positive $D$. Combining (4.8) and (4.9) yields

$$
\left\|\sigma_{B}\left(x_{0}\right)-\sigma_{A}\left(x_{0}\right)\right\|=\left\|w_{0}\right\| \leqslant \gamma \exp \left((\eta-\delta) / k\left|x_{0}\right|\right),
$$

with

$$
\gamma=D \exp ((-\eta+\delta) / \varepsilon k) \cdot \max \left\{\left\|\sigma_{B}(\xi)-\sigma_{A}(\xi)\right\|:\|\xi\|=\varepsilon\right\} .
$$

The estimate (4.10) is of the desired form (4.4).

Case 2. $\left\|x\left(t ; x_{0}\right)\right\|<\varepsilon$ for all $t \leqslant 0$. Then instead of (4.9) we have

$$
\left\|w_{0}\right\| \leqslant D\left\|w\left(t ; x_{0}\right)\right\| \exp (\delta-\eta) t
$$

for all $t \leqslant 0$; moreover,

$$
\left\|w\left(t ; x_{0}\right)\right\| \leqslant \max \left\{\left\|\sigma_{B}(\xi)-\sigma_{A}(\xi)\right\|:\|\xi\| \leqslant \varepsilon\right\} .
$$

We conclude that $w_{0} \equiv 0$, so $\sigma_{A}\left(x_{0}\right)=\sigma_{B}\left(x_{0}\right)$ and (4.10) (or (4.4)) is trivially satisfied.

Both points of view, i.e. the first one using (4.3) and the second one using (4.8) and (4.9), have now been discussed heuristically. In the proofs of Lemma 4.1 and Theorem 4.2 the respective treatments will be made precise.

Lemma 4.1. Consider the dynamical system

$$
d x / d t=L x+g(x)
$$

on the set $D_{r}=\left\{x \in \mathbf{R}^{n}:\|x\| \leqslant r\right\}$. Here $L$ is linear, $\operatorname{Re}(\operatorname{spec}(L))=0, g$ is continuous from $D_{r}$ to $\mathbf{R}^{n}$ and, for some positive $K$,

$$
\max _{x \in D_{r}} \frac{\|g(x)\|}{\|x\|^{2}}=K<\infty
$$

By $x(t ; \xi)$ we denote the solution of $(4.14)$ with $x(0 ; \xi)=\xi$; here $\xi \in D_{r}$. Let $p$ and $c$ be such that

$$
\left\|e^{L t}\right\| \leqslant(1+c|t|)^{p} \text { for all } t,-\infty<t<\infty .
$$


Define the function $f(t, \xi): I \times R^{n} \rightarrow \mathbf{R}$ by

$$
f(t, \xi)=\frac{(1-c t)^{p}\|\xi\|}{1-(K /(2 p+1) c)\|\xi\|\left\{(1-c t)^{2 p+1}-1\right\}} \quad(\|\xi\| \neq 0)
$$

here $I$ is the largest interval of the form $(-M, 0]$ on which this function is continuous. For every $\xi \in D_{r}$ the (nonpositive) number $t_{0}(\xi, r)$ denotes the solution of

$$
f\left(t_{0}(\xi, r), \xi\right)=r .
$$

Then:

(i) For all $t, t_{0}(\xi, r) \leqslant t \leqslant 0$, we have $\|x(t ; \xi)\| \leqslant f(t ; \xi)$.

(ii) There are positive numbers $\eta<r$ and $D$ such that

$$
\|\xi\|<\eta \Rightarrow\left|t_{0}(\xi, r)\right|>D /\|\xi\|^{1 /(2 p+1)} \text {. }
$$

(iii) For all $\xi \in D_{r}$ and $t>0$ for which $x(t ; \xi) \in D_{r}$ we have

$$
\|x(t ; \xi)\| \geqslant\left(\frac{K}{(2 p+1) c}\left\{(1+c t)^{2 p+1}-1\right\}+\frac{(1+c t)^{p}}{\|\xi\|}\right)^{-1} \quad(\|\xi\| \neq 0) .
$$

The proof of Lemma 4.1 is postponed to $\$ 8.1$.

REMARKS. 1. Lemma 4.1(i) and (4.18) imply that $x(t ; \xi)$ stays within a distance $r$ from the origin for $t_{0}(\xi, r) \leqslant t \leqslant 0$.

2. The estimate in (ii) is sharp. An example with $p=0$ is the differential equation $\dot{x}=-x^{2}(x \in \mathbf{R})$ with solution $x=\xi(1+\xi t)^{-1} ;$ here $t_{0}(\xi ; r)=-\left(r^{-1}+\xi^{-1}\right)=$ $O_{s}\left(\xi^{-1}\right)$.

An example for more general $p$ is the system in $\mathbf{R}^{p+1}$ :

$$
\begin{aligned}
& d x_{i} / d t=-x_{i+1} ; \quad x_{i}(0)=0 \quad(i=1, \ldots, p), \\
& d x_{p+1} / d t=-x_{1}^{2} ; \quad x_{p+1}(0)=\xi .
\end{aligned}
$$

If we perform a scaling transformation

$$
x_{i}=\xi^{(p+i) /(2 p+1)} y_{i} ; \quad t=-\xi^{-1 /(2 p+1)} \tau,
$$

then

$$
\begin{aligned}
& d y_{i} / d \tau=y_{i+1} ; \quad y_{i}(0)=0 \quad(i=1, \ldots, p), \\
& d y_{p+1} / d \tau=y_{1}^{2} ; \quad y_{p+1}(0)=1 .
\end{aligned}
$$

The solution of (4.22) will reach infinity in finite time, say $T_{p}$, e.g.,

$$
T_{2}=\int_{0}^{\infty}\left\{\frac{2}{3} z^{3}+1\right\}^{-1 / 2} d z
$$

This implies that, for every $r>0,\left|t_{0}(\xi, r)\right|<\xi^{-1 /(2 p+1)} T_{p}$.

In the case that $g(x)$ approaches 0 faster than $\|x\|^{2}$ the estimates can be improved; this also carries over to the following theorem.

TheOREM 4.2. We consider system (3.16). For $B_{i}, L_{i}, n_{i}(i=1,2)$ we make the assumptions $\left(\mathrm{A}_{\mathrm{i}-\mathrm{iv}}\right)$ of $\S 2$; let $n_{i} \in C^{1}$. Let $S_{A}$ and $S_{B}$ denote two $C^{1}$ center manifolds $S_{\alpha}=\left\{\left(x, \sigma_{\alpha}(x)\right): x \in U\right\}(\alpha=A, B)$. Here $U$ is some neighborhood of 0 in $B_{1}$. 
(i) If $\operatorname{Re}\left(\operatorname{spec}\left(L_{1}\right)\right)=0$ and the algebraic and geometric multiplicities of the eigenvalues of $L_{1}$ are equal, then there are constants $D_{1}, D_{2}$ and a neighborhood $U$ of 0 in $B_{1}$ such that

$$
\left\|\sigma_{A}(x)-\sigma_{B}(x)\right\|_{B_{2}} \leqslant D_{1} \exp \left(-D_{2} /\|x\|_{B_{1}}\right) .
$$

(ii) If $\left\|e^{L_{-1} t}\right\|_{B_{1}} \leqslant(1+c|t|)^{p}$ for some $p \geqslant 1$, then there are constants $D_{1}$ and $D_{2}$ and a neighborhood $U$ of 0 in $B_{1}$ such that

$$
\left\|\sigma_{A}(x)-\sigma_{B}(x)\right\|_{B_{2}} \leqslant D_{1} \exp \left(-D_{2} /\|x\|_{B_{1}}^{1 /(2 p+1)}\right) .
$$

Theorem 4.2 is proved in $\$ 8.2$.

REMARK. These estimates are sharp; we are able to construct examples using the remark following Lemma 4.1.

Corollary 4.3. Consider the system (3.11); suppose $n_{i} \in C^{k+2}$ with $k \geqslant 0$. Let $U$ be a neighborhood of 0 in $B_{1}$ and let $\sigma_{A}$ and $\sigma_{B}$ satisfy the following conditions:

(i) $\sigma_{A} \in C^{k+2}\left(U \rightarrow B_{2}\right)$ and $\left\{\left(x, \sigma_{A}(x)\right): x \rightarrow U\right\}$ is a center manifold of (3.11).

(ii) $\sigma_{B} \in C^{k+2}\left(\stackrel{\circ}{U} \rightarrow B_{2}\right) \cap C^{0}\left(U \rightarrow B_{2}\right)$ and $\left\{\left(x, \sigma_{B}(x)\right): x \in U\right\}$ is an invariant manifold of (3.11). Here $\stackrel{\circ}{U}=U \backslash\{0\}$.

Then $\sigma_{B} \in C^{k+1}\left(U \rightarrow B_{2}\right)$ and for $\varepsilon$ small enough there are constants $D_{1}$ and $D_{2}$ such that

$$
\|x\| \leqslant \varepsilon \Rightarrow\left\|(d / d x)^{k+1}\left(\sigma_{A}(x)-\sigma_{B}(x)\right)\right\| \leqslant D_{1} \exp \left(-D_{2} /\|x\|^{1 /(2 p+1)}\right) .
$$

Here $p$ is the number defined in Theorem 4.2(ii).

Corollary 4.3 is proved in $\$ 8.3$.

Remark. The estimate (4.25) shows that the Taylor series of two center manifolds are identical. There are many other ways to illustrate this fact. For instance, differentiation of $\sigma\left(u_{1}(t)\right)$ with respect to $t$ gives, with (3.11),

$$
\left(d \sigma / d u_{1}\right)\left(L_{1} u_{1}+n_{1}\left(u_{1}, \sigma\left(u_{1}\right)\right)\right)=L_{2} \sigma\left(u_{1}\right)+n_{2}\left(u_{1}, \sigma\left(u_{1}\right)\right)
$$

(see also $[4,18]$ ).

Introducing a polynomial approximation

$$
\sigma_{N}\left(u_{1}\right)=\sum_{n=2}^{N} f_{n}\left(u_{1} ; \cdots ; u_{1}\right),
$$

where the $f_{n}$ are homogeneous polynomials of degree $n$, and then equating corresponding powers of $u_{1}$ we obtain a unique formal Taylor expansion of $\sigma$; for a discussion of this procedure as well as the numerical implementation, see $[15,16,23]$. The number $N$ can be taken as high as the differentiability of $n_{i}$ permits $(i=1,2)$. The polynomials $\sigma_{N}(N \geqslant 2)$ form a sequence of asymptotic approximations of the bundle of center manifolds. The domain of validity may shrink to zero for increasing $N$; for an example of this behaviour see $\$ 1$.

5. Differentiability. In $\S 2$ we have treated an example (2.37) of a dynamical system which possessed a $C^{k}$ center manifold for all $k$, but no $C^{\infty}$ center manifold. For an earlier example, see [46]. 
In this section we shall investigate a class of problems where this situation does not occur and where the existence of a $C^{\infty}$ center manifold is guaranteed.

We shall treat separately the cases $\operatorname{dim} B_{1}>1$ (Theorem 5.1) and $\operatorname{dim} B_{1}=1$ (Theorem 5.2).

THEOREM 5.1. Consider the system

$$
\begin{array}{ll}
d x / d t=L_{1} x+n_{1}(x, y), & x \in B_{1}, \\
d y / d t=L_{2} y+n_{2}(x, y), & y \in B_{2} .
\end{array}
$$

Here $B_{i}, L_{i}$ and $n_{i}(i=1,2)$ satisfy the assumptions $\left(\mathrm{A}_{\mathrm{i}-\mathrm{iv}}\right)$ of $\$ 2$; we also assume that $1<\operatorname{dim} B_{1}<\infty$ and that $n_{i}$ is in $C^{\infty}(i=1,2)$.

Define the function $F(x, y)$ by

$$
F(x, y)=\left(x, L_{1} x+n_{1}(x, y)\right),
$$

where $(\cdot, \cdot)$ denotes a scalar product on the finite-dimensional space $B_{1}$. If there is a neighborhood $U \times V$ of $(0,0) \in B_{1} \times B_{2}$ such that $F(x, y)$ does not vanish on $U \times V$ except for $x=0$, then (5.1) has a $C^{\infty}$ center manifold.

REMARK 1. The condition $F(x, y)>0$ implies that $(0,0)$ is an attractor for $t \rightarrow-\infty ; F(x, y)<0$ means that $(0,0)$ is an attractor for $t \rightarrow+\infty$.

REMARK 2. The requirement that $F$ does not vanish except for $x=0$ implies that the stable manifold of (5.1) coincides with $B_{2}$. This is no restriction because a $C^{\infty}$ coordinate transformation always exists which brings the stable manifold of (5.1) into this position.

Proof of Theorem 5.1. (i) We first study the case $F(x, y)>0$. Let $\{(x, \sigma(x))$ : $\left.x \in U_{0}\right\}$ be a $C^{1}$ center manifold of (5.1); assume $U_{0} \subset U$. By Theorem 3.2 and the remark made above this center manifold is unique, and for this one function $\sigma$ there are open neighborhoods $U_{k} \subset U_{0}(k \geqslant 1)$ such that $\sigma \in C^{k}\left(U_{k} \rightarrow B_{2}\right)$. Let $x_{0} \in U_{0}$ be arbitrary. If $(x(t ; \xi), y(t ; \xi))$ denotes the solution of the initial value problem (5.1) with initial value $x(0 ; \xi)=\xi, y(0 ; \xi)=\sigma(\xi)$, then there is a point $x_{k} \in U_{k}$ and a number $t_{k}>0$ such that $x\left(t_{k} ; x_{k}\right)=x_{0}$. Because $\sigma$ is $C^{k}$ in an open neighborhood $E_{k} \subset U_{k}$ of $x_{k}$, the set

$$
\left\{\left(x\left(t_{k} ; \xi\right), y\left(t_{k} ; \xi\right)\right): \xi \in E_{k}\right\}
$$

is a $C^{k}$ manifold. But this set represents the center manifold in a neighborhood of $\left(x_{0}, \sigma\left(x_{0}\right)\right)$. So the center manifold is $C^{k}$ on all of $U_{0}$.

(ii) Now we study $F(x, y)<0$, in which case there is not a unique center manifold available. Suppose that the ball with radius $a_{3}$ in $B_{1}$ is contained in $U$. Define a function $\phi \in C^{\infty}([0, \infty) \rightarrow \mathbf{R})$ such that there are numbers $0<a_{1}<a_{2}<$ $a_{3}$ with

$$
\begin{gathered}
\phi(x)=1 \quad \text { for } 0 \leqslant x \leqslant a_{1}, \\
\phi(x)>0 \quad \text { for } a_{1} \leqslant x<a_{2}, \\
\phi^{\prime}\left(a_{2}\right)<0, \quad \phi\left(a_{2}\right)=0 \\
\phi(x)=0 \quad \text { for } x \geqslant a_{3} .
\end{gathered}
$$


One proves with the techniques of $\$ 2$ that there is a unique global $C^{1}$ center manifold $S=\left\{(x, \sigma(x)): x \in B_{1}\right\}$ of

$$
d x / d t=\left\{L_{1} x+n_{1}(x, y)\right\} \cdot \phi(\|x\|), \quad d y / d t=L_{2} y+n_{2}(x, y) \cdot \phi(\|x\|) .
$$

The set $I=\left\{(x, y) \in B_{1} \times B_{2}:\|x\|=a_{2}\right.$ and $\left.y=0\right\}$ is an invariant manifold of (5.5). Moreover, the spectrum of the flow linearized at $\left(x_{0}, 0\right) \in I$ consists of the union of $\operatorname{spec}\left(L_{2}\right)$ and the collection of eigenvalues of the operator

$$
\mathscr{L} \xi=\left\{L_{1} x_{0}+n_{1}\left(x_{0}, 0\right)\right\}\left(\phi^{\prime}\left(a_{2}\right) / a_{2}\right)\left(x_{0}, \xi\right) .
$$

The equation $\mathscr{L} \xi=\lambda \xi$ leads to

$$
F\left(x_{0}, 0\right) \phi^{\prime}\left(a_{2}\right) / a_{2}=\lambda
$$

for all $\xi \in B_{1}$ which are not tangent to $I$. By (5.4c) the $\lambda$ which satisfy (5.7) are positive, and we conclude that $I$ is an hyperbolic invariant manifold of (5.5) and that $\sigma$ is the unstable manifold of (5.5) at $I$, which implies that $\sigma$ is $C^{\infty}$ in a neighborhood of $I$ [13]. Using an argument as in (i) we conclude that $\sigma \in C^{\infty}\left(\stackrel{\circ}{U} \rightarrow B_{2}\right)$, where $\stackrel{U}{U}$ is the ball of radius $a_{2}$ around 0 in $B_{1}$, with 0 deleted from it.

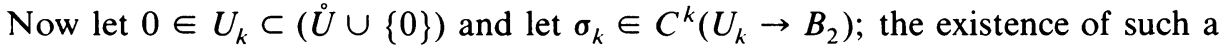
pair $U_{k}, \sigma_{k}$ for all $k \geqslant 0$ is guaranteed by Theorem 2.1. We use Corollary 4.3 to

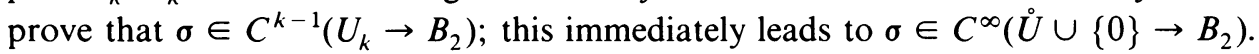
The restriction of $\sigma$ to the ball of radius $a_{1}$ represents the $C^{\infty}$ center manifold of (5.1).

THEOREM 5.2. Consider system (5.1) with the assumptions $\left(\mathrm{A}_{\mathrm{i}-\mathrm{iv}}\right)$ of $\$ 2$. Suppose $\operatorname{dim} B_{1}=1$ and $n_{i} \in C^{\infty}$. Let $\Sigma$ denote a $C^{1}$ center manifold of (5.1) defined on $a$ neighborhood $U_{0}$ of 0 in $B_{1}$ :

$$
\Sigma=\left\{(x, \sigma(x)): x \in U_{0}\right\} .
$$

Define the function $f: U_{0} \rightarrow B_{1}$ by $f(x)=n_{1}(x, \sigma(x))$. If there is a $U_{1} \subset U_{0}$ such that $f$ has no zeros in $U_{1}$ except at the origin, then there is a $U_{2} \subset U_{1}$ such that $\sigma \in$ $C^{\infty}\left(U_{2} \rightarrow B_{2}\right)$.

Proof. Let the interval $\left[0, a_{3}\right) \subset U_{1}$. If $f>0$ on $\left(0, a_{3}\right)$, then the part of $\Sigma$ above $\left[0, a_{3}\right)$ is unique and, by the proof of Theorem 5.1(i), we find $\sigma \in C^{\infty}\left(\left[0, a_{3}\right)\right)$.

If $f<0$ on $\left(0, a_{3}\right)$, then we modify the system (5.1) by a $C^{\infty}$ function $\phi$ which satisfies (5.4). The modified system (5.5) will have a unique global $C^{1}$ center manifold $\Sigma^{*}=\left\{\left(x, \sigma^{*}(x)\right): x \in B_{1}\right\}$ and 0 is an attractor for $t \rightarrow \infty$ in $\left.\Sigma^{*}\right|_{\left[0, a_{3}\right)}$. Let $x_{1}$ be the $x$-coordinate of the first singular point of (5.5) which lies to the right of 0 in $\Sigma^{*}: 0<x_{1} \leqslant a_{2}$ (the point $x_{1}$ not necessarily coincides with $a_{2}$; see e.g. (3.5), (3.6)). Then the restriction of $\sigma$ to $\left[0, x_{1}\right]$ is either the unstable manifold of (5.5) at $x_{1}$ or it is the center manifold at $x_{1}$ with the additional property that, for $t \rightarrow-\infty, x_{1}$ is an attractor. By the proof of Theorem 5.1(i) we find that $\sigma \in C^{\infty}\left(\left(0, x_{1}\right] \rightarrow B_{2}\right)$ and by the proof of Theorem 5.1(ii) we conclude $\sigma \in C^{\infty}\left(\left[0, x_{1}\right] \rightarrow B_{2}\right)$.

On the interval $\left(-a_{3}, 0\right]$ the arguments are similar.

Corollary 5.3 (due to F. Dumortier). Let $\sigma$ and $f$ be as in Theorem 5.2; suppose $\sigma \in C^{k}\left(U_{0} \rightarrow B_{2}\right)$ and $f=x^{p} \tilde{f}(x), p \leqslant k, \tilde{f}(0) \neq 0$. Then for a neighborhood $U_{2}$ of 0 with $U_{2} \subset U_{0}$ we have $\sigma \in C^{\infty}\left(U_{2} \rightarrow B_{2}\right)$. 
THEOREM 5.4. Consider system (5.1) with the assumptions $\left(\mathrm{A}_{\mathrm{i}-\mathrm{iv}}\right)$ of $\S 2$, let $n_{i} \in C^{\infty}$ and suppose $\operatorname{dim} B_{1}=2$ and $\operatorname{spec}\left(L_{1}\right)=\{ \pm i\}$. Let $\Sigma$ denote a $C^{1}$ center manifold of (5.1) defined on a neighborhood $U_{0}$ of 0 in $B_{2}$. If there is a $U_{1} \subset U_{0}$ such that the restriction of $\Sigma$ to $U_{1}$ contains no periodic solutions of (5.1) (except the origin), then there is a $U_{2} \subset U_{1}$ such that $\Sigma$ is $C^{\infty}$ on $U_{2}$.

The proof of this theorem is analogous to the proof of Theorem 5.2.

The main requirement in Theorems 5.2 and 5.4 is the condition that 0 is an isolated stationary point or periodic solution. The following example shows the necessity of these conditions.

EXAmple. Consider the $C^{\infty}$ dynamical system

$$
\begin{aligned}
& \dot{x}=e^{-1 / x^{2}} \sin x^{-1} \quad(x \in \mathbf{R}), \\
& \dot{y}=-y \quad(y \in \mathbf{R}) .
\end{aligned}
$$

The unique center manifold is

$$
\Sigma=\{(x, 0): x \in \mathbf{R}\} .
$$

The manifold $\Sigma$ contains a sequence of stationary points $x_{n}=(n \pi)^{-1}$; the points $x_{2 n}$ are nodes with eigenvalues $\lambda_{1}^{(n)}=-1$ and $\lambda_{2}^{(n)}=-n^{2} \pi^{2} \exp \left(-n^{2} \pi^{2}\right)$. By making a small $C^{\infty}$ modification of (5.9) in the region $\{(x, y): 1 / 2 n<x<1 /(2 n+1)\}$ we can achieve that a discontinuity arises in the derivative of order $\lambda_{1}^{(n)} / \lambda_{2}^{(n)}$ of the center manifold of the modified system. This manipulation produces another $C^{\infty}$ dynamical system without a $C^{\infty}$ center manifold.

By supplying (5.9) with an extra equation $\dot{\psi}=1$ and regarding $(x, \psi)$ as polar coordinates in $B_{1}$, we obtain a counterexample for the case $\operatorname{dim} B_{1}=2$.

REMARK 1. In the case that $\operatorname{dim} B_{1}=1$ we now summarize Corollary 5.3 and the example above. Let $n_{1}$ and $n_{2}$ be $C^{\infty}$ and let $\sigma(x)$ represent a $C^{k}$ center manifold of $(5.1), k \geqslant 1$.

(i) If the $p$ th order $(p \leqslant k)$ derivative of $n_{1}(x, \sigma(x))$ does not vanish at $x=0$, then $\sigma$ is $C^{\infty}$.

(ii) There is an example of a dynamical system with a center manifold defined by a function $\sigma$ which is not $C^{\infty}$ in a neighborhood of 0 , and where all derivatives of $n_{1}(x, \sigma(x))$ exist and are zero.

In the analytic category (i.e., $n_{1}$ and $n_{2}$ are analytic) the situation is almost opposite:

(iii) There is a dynamical system where $n_{1}(x, \sigma(x))$ has a nonvanishing 3rd derivative at $x=0$, but there is no analytic center manifold. Take for example

$$
\dot{x}=x^{3}, \quad \dot{y}=-y+x^{2} .
$$

(iv) If all derivatives of $n_{1}(x, \sigma(x))$ vanish at $x=0$, then $\sigma$ is analytic. This follows from the fact that $\sigma(x)$ satisfies

$$
n_{1}(x, \sigma(x))(d \sigma / d x)=L_{2} \sigma+n_{2}(x, \sigma(x)) .
$$

Because the (formal) power series of $n_{1}(x, \sigma(x))$ is zero, we have

$$
L_{2} \sigma+n_{2}(x, \sigma(x))=0,
$$


which by the implicit function theorem yields a unique analytic function $\sigma(x)$. The center manifold is filled up with stationary solutions.

Theorem 5.4 and the subsequent example show that (i) and (ii) also hold for $\operatorname{dim} B_{1}=2$; in that case (iii) is obvious. The analogue of (iv) is treated in [3]; for more details on two-dimensional manifolds filled with periodic solutions, see [29] and $§ 7$.

REMARK 2. The cut-off function in the proof of Theorem 5.2 may introduce a new singular point $x_{1}$ (compare (3.5), (3.6)). If $\operatorname{dim} B_{1}$ is higher than 1 , it becomes increasingly difficult to deal with these uncontrollable singular points and other attractors.

We have prevented this difficulty by strengthening the condition on the flow in a single center manifold (in Theorem 5.2) to a condition on the flow in a full neighborhood of 0 in $B_{1} \times B_{2}$ (in Theorem 5.1).

However, this stronger assumption is quite restrictive: for instance, example (3.5), (3.6) fits into Theorem 5.2 but not into Theorem 5.1.

REMARK 3. Generally, an analytic dynamical system does not have an analytic center manifold.

For counterexamples, see [28] and (2.37). In a special situation, however, a two-dimensional center manifold related to a pair of imaginary eigenvalues may be analytic (see Theorem 7.1).

6. Analytic manifolds containing periodic solutions. In this section we consider again the equation on the Banach space $B$ :

$$
d u / d t=L u+N(u) .
$$

Here $L$ is linear and $N(u)$ can be written as a convergent power series without constant or linear terms.

In his celebrated paper [29] Lyapunov analyzed (6.1) in the case that $L$ has a simple pair of eigenvalues $\pm i$. Under certain conditions (mainly the presence of an integral and a nonresonance condition; for details see \$7) Lyapunov proved the existence of a two-dimensional manifold tangent to the eigenspace belonging to the eigenvalues $\pm i$; this manifold is filled with an analytic one-parameter family of periodic solutions.

If the operator $L$ would have no purely imaginary eigenvalues except for $\pm i$, then in our present framework we would call Lyapunov's manifold a center manifold. However, the occurrence of the analytic invariant manifold filled with periodic solutions is not sensitive to the presence of other imaginary eigenvalues provided a nonresonance condition is satisfied. The invariant manifold of Lyapunov then is a submanifold of the center manifold and therefore will be called a subcenter manifold (see $\$ 7$ for definition).

Lyapunov constructs his manifold in [29] by proving the convergence of its power series representation. In $\S \S 6$ and 7 we shall construct analytic manifolds which contain all periodic solutions of prescribed period (Theorem 6.3); these manifolds are not necessarily invariant. The proof is based on the implicit function theorem. In $\$ 7$ we shall analyze the case that these manifolds are two-dimensional while (6.1) 
possesses an integral: the manifold is then invariant and filled by periodic solutions and a new proof of Lyapunov's theorem is obtained (Theorem 7.1). We conclude $\S 7$ by studying subcenter manifolds in more detail.

We now state the assumptions on (6.1) which we shall make in $\$ \S 6$ and 7.

$\mathrm{H}_{1} . B$ can be decomposed into $B=B_{1} \oplus B_{2}$, where $L\left(B_{i}\right) \subset B_{i}(i=1,2)$. There exist projection operators $P_{1}$ and $P_{2}$ such that $P_{i} B=B_{i}$ and $P_{1}+P_{2}=1$.

$\mathrm{H}_{2} \cdot \operatorname{dim} B_{1}<\infty, \operatorname{Re}\left(\operatorname{spec}\left(\left.L\right|_{B_{1}}\right)\right)=0,0 \notin \operatorname{spec}\left(\left.L\right|_{B_{1}}\right)$ and

$$
\exp (L) P_{1}=P_{1} \text {. }
$$

$\mathrm{H}_{3} . \operatorname{dim} B_{2}<\infty$ and the operator $K: B_{2} \rightarrow B_{2}$ defined by

$$
K=(\exp (L)-1) i_{2}
$$

has a bounded inverse. Here $i_{2}$ is the identity $B_{2} \hookrightarrow B$.

REMARK. The hypothesis $\mathrm{H}_{3}$ does not exclude the possibility that the spectrum of $\left.L\right|_{B_{2}}$ contains imaginary points; however, the assumption that $K$ is invertible does not allow these eigenvalues to be integer multiples of $2 \pi i$.

We assume that $B_{2}$ is finite-dimensional because most of the applications of this section lie in the field of ordinary differential equations. The results stated below can be extended to infinite-dimensional spaces $B_{2}$ by assuming that $L$ generates a differentiable semigroup for $t>0$ and taking into account the remark following equation (2.9).

We now state two theorems which give some insight into the general behaviour of families of periodic solutions near equilibrium points; Theorem 6.2 is needed for the proof of Theorem 6.3; Theorem 6.1 will be used in $\$ 7$ but it is more natural to formulate it here.

THEOREM 6.1. Consider the equation (6.1) where $L$ and $B$ satisfy hypotheses $H_{1}$ and $\mathrm{H}_{3}$, and the spectrum of $\left.L\right|_{B_{2}}$ contains no points on the imaginary axis. Suppose that $\operatorname{dim} B_{1}<\infty$ and that the spectrum of $\left.L\right|_{B_{1}}$ consists of the points $\pm 2 \pi i \nu_{1}^{-1}, \ldots, \pm 2 \pi i \nu_{p}^{-1}$ while these eigenvalues all have equal algebraic and geometric multiplicities. Suppose that $N$ is continuously differentiable and that $N(0)=0, N^{\prime}(0)=0$. Let $u_{\lambda}$ be a family of periodic solutions of the nonlinear equations with period $T_{\lambda} ; \lambda$ is in a set $\left\{\lambda \in \mathbf{R}^{n}\right.$ : $0<|\lambda|<r\}$. Suppose that $u_{\lambda}$ depends continuously on $\lambda$ and $\left\|\left|u_{\lambda}\right|\right\|$ approaches 0 for $\lambda \rightarrow 0$, where

$$
\left\|u_{\lambda}\right\|\left\|=\max _{0 \leqslant t<T_{\lambda}}\right\| u_{\lambda}(t) \|
$$

(i) If $T_{\lambda}$ is bounded, then there is an integer $j_{0}, 1 \leqslant j_{0} \leqslant p$, such that the distance between $T_{\lambda}$ and the set $\left\{n \nu_{j_{0}}: n\right.$ an integer $\}$ approaches 0 when $\lambda$ tends to 0 .

(ii) If $T_{\lambda}$ is continuous for $\lambda \neq 0$ and $\left\|u_{\lambda}\right\| T_{\lambda}$ approaches 0 when $\lambda$ tends to 0 , then there are integers $n_{0}$ and $j_{0}$ such that $T_{\lambda}$ converges to $n_{0} \nu_{j_{0}}$ for $\lambda$ approaching 0 .

(iii) If there are positive real numbers $k$ and $c$ such that

$$
\left|n \nu_{i}-m \nu_{j}\right| \geqslant c(\max (n, m))^{-k}
$$

for every rationally independent pair $\nu_{i}, \nu_{j}$ and every pair of integers $n, m$, and if there is a real number $l, l>k+1$, such that $T_{\lambda}^{l}\left\|\left|u_{\lambda} \|\right|\right.$ is bounded, then the conclusions of (i) remain valid. 
The proof of this theorem is a little technical and it is deferred to $§ 8.4$.

REMARK. Theorem 6.1 studies families of periodic solutions which are continuously parametrized by $\lambda$ (e.g. a point on the orbit depends continuously on $\lambda$ ). In (ii) the period $T_{\lambda}$ also is continuous in $\lambda$, while in (i) and (iii) discontinuous changes in this period are allowed (for example by period-doubling bifurcations).

THEOREM 6.2. Let $L_{i}, B_{i}$ satisfy hypotheses $\mathrm{H}_{1}-\mathrm{H}_{3}$ and let $N$ be continuously differentiable. Let $u_{\lambda}$ be a family of periodic solutions of (6.1) as in Lemma 6.1. Suppose that $T_{\lambda}$ approaches 1 for $\lambda$ approaching 0 . Then the family $u_{\lambda}$ is tangent to $B_{1}$ in the origin.

Proof. Define $\varepsilon_{\lambda}=\left\|u_{\lambda}\right\| \mid ; w_{\lambda}=\varepsilon_{\lambda}^{-1} u_{\lambda}$ (we exclude those $\lambda$ for which $\varepsilon_{\lambda}=0$ ). Then

$$
d w_{\lambda} / d t=L w_{\lambda}+\varepsilon_{\lambda} g\left(w_{\lambda}, \varepsilon_{\lambda}\right)
$$

where

$$
g(y, \varepsilon)=\varepsilon^{-2} N(\varepsilon y)
$$

So,

$$
w_{\lambda}(0)=w_{\lambda}\left(T_{\lambda}\right)=e^{L T_{\lambda}} w_{\lambda}(0)+\varepsilon_{\lambda} \int_{0}^{T_{\lambda}} e^{L\left(T_{\lambda}-\tau\right)} g\left(w_{\lambda}(\tau), \varepsilon_{\lambda}\right) d \tau
$$

which implies

$$
\left(1-e^{L T_{\lambda}}\right) w_{\lambda}(0)=O\left(\varepsilon_{\lambda}\right) .
$$

From hypothesis $\mathrm{H}_{3}$ we conclude that $P_{2} w_{\lambda}(0)=O\left(\varepsilon_{\lambda}\right)$, hence

$$
P_{2} u_{\lambda}(t)=O\left(\varepsilon_{\lambda}^{2}\right) \quad\left(0 \leqslant t<T_{\lambda}\right),
$$

which proves the theorem.

THEOREM 6.3. Consider system (6.1). In addition to the hypotheses $\mathrm{H}_{1}-\mathrm{H}_{3}$ we suppose that $N(u)$ is analytic. Then an analytic manifold $A \subset B$ exists with the properties

(i) $\operatorname{dim} A=\operatorname{dim} B_{1}$ and in the origin $A$ is tangent to $B_{1}$,

(ii) every sufficiently small periodic solution with period sufficiently close to 1 lies entirely in $A$,

(iii) there is an analytic function $v: A \rightarrow B_{1}$ such that $v$ is zero exactly on the periodic solutions from (ii).

Proof. Put $u=\varepsilon z$; with (6.1) and (6.7) we obtain

$$
d z / d t=L z+\varepsilon g(z, \varepsilon) .
$$

For every $a \in B_{1}, b \in B_{2}$ and $\varepsilon>0$, denote by $z(a, b, \varepsilon ; t)$ the solution of (6.11) which satisfies $z(a, b, \varepsilon ; 0)=a+b$. By taking $a, b, \varepsilon$ small enough, this solution exists for arbitrarily long positive time intervals.

Define

$$
\begin{aligned}
U & =\left\{a \in B_{1}: r_{1}<|a|<r_{2}\right\} \\
C_{T} & =C^{0}(U \rightarrow \mathbf{R}), \quad C_{b}=C^{0}\left(U \rightarrow B_{2}\right) .
\end{aligned}
$$


We now study the operator $\Gamma$,

$$
\Gamma: C_{b} \times C_{T} \times \mathbf{R} \rightarrow C_{b} \times C_{T},
$$

given by

$$
(\Gamma(b, T ; \varepsilon))(a)=\left(\begin{array}{c}
P_{2}(z(a, b(a), \varepsilon ; T(a))-(a+b(a))) \\
\left(P_{1} z(a, b(a), \varepsilon ; T(a)), L_{1} a\right)
\end{array}\right) .
$$

Here $(\cdot, \cdot)$ denotes the standard scalar product on $B_{1}=\mathbf{R}^{n}$. Clearly, $\Gamma$ depends analytically on $b, T$ and $\varepsilon . \Gamma(0,1 ; 0)=0$ and

$$
\operatorname{grad}_{b, T} \Gamma(0,1 ; 0)=\left(\begin{array}{cc}
K & 0 \\
0 & \left|L_{1} a\right|^{2}
\end{array}\right) \text {. }
$$

This derivative is a linear operator from $C_{b} \times C_{T}$ to $C_{b} \times C_{T}$ and by hypothesis $\mathrm{H}_{3}$ it is boundedly invertible. The implicit function theorem now yields a unique analytic one-parameter family $b_{\varepsilon} \subset C_{b}, T_{\varepsilon} \subset C_{T}$ such that

$$
\Gamma\left(b_{\varepsilon}, T_{\varepsilon} ; \varepsilon\right)=0 \text { on } U \text {. }
$$

The analyticity of $b_{\varepsilon}, T_{\varepsilon}$ as functions of $\varepsilon$ implies the convergence on $\left[0, \varepsilon_{0}\right] \times U$ of the series

$$
b_{\varepsilon}(a)=\sum_{n=2}^{\infty} \varepsilon^{n-1} b_{n}(a), \quad T_{\varepsilon}(a)=\sum_{n=0}^{\infty} \varepsilon^{n} T_{n}(a) .
$$

Here $b_{n}(a) \in C_{b}$ and $T_{n}(a) \in C_{T}$ for all $n$. The explicit expressions for $b_{n}$ and $T_{n}$ are obtained by inserting (6.17) into (6.14) and comparing powers of $\varepsilon$ in the equation $\Gamma=0$. However, using (6.7) one easily proves that $b_{n}(a)$ and $T_{n}(a)$ are homogeneous of order $n$ in their argument $a$, so we are able to define functions $\beta$ and $\tau$ by

$$
\begin{gathered}
\beta(\varepsilon a)=\varepsilon b_{\varepsilon}(a)=\sum_{n=2}^{\infty} b_{n}(\varepsilon a), \\
\tau(\varepsilon a)=T_{\varepsilon}(a)=\sum_{n=0}^{\infty} T_{n}(\varepsilon a) .
\end{gathered}
$$

Both $\beta$ and $\tau$ are defined on $V=\left\{a \in B_{1}:|a|<\varepsilon_{0} r_{2}\right\}$, and their series expansion (6.18) converges. The manifold $A=\{x+\beta(x): x \in V\}$ is analytic and satisfies property (i).

Let $u$ be a small periodic solution of (6.1) with period $T$ close to 1 . Choose $a_{0} \in\left(0, \varepsilon_{0} r_{2}\right)$ and define

$$
\begin{aligned}
& \varepsilon=a_{0}^{-1} \max _{0 \leqslant t<T}\|u(t)\|, \quad w=\varepsilon^{-1} u, \\
& \tilde{\alpha}=P_{1} w(0) \quad \text { and } \tilde{\beta}=P_{2} w(0) .
\end{aligned}
$$

The scaled function $w$ will satisfy (6.6). Because $w$ is periodic with period $T$,

$$
P_{2}(w(\tilde{\alpha}, \tilde{\beta}, \varepsilon ; T)-(\tilde{\alpha}+\tilde{\beta}))=0, \quad\left(P_{1} w(\tilde{\alpha}, \tilde{\beta}, \varepsilon ; T), L_{1} \tilde{\alpha}\right)=0 .
$$

As in the proof of Theorem 6.2 we conclude that $\tilde{\beta}$ is small for $u$ small and $T$ close to 1. From $\tilde{\alpha} \approx a_{0}<\varepsilon_{0} r_{2}$ and the uniqueness of the solution $b_{\varepsilon}(a), T_{\varepsilon}(a)$ of $(6.16)$ on $0 \leqslant a<\varepsilon_{0} r_{2}$, we conclude

$$
b_{\varepsilon}(\tilde{\alpha})=\tilde{\beta} \quad \text { and } \quad T_{\varepsilon}(\tilde{\alpha})=T,
$$

$$
\varepsilon(\tilde{\boldsymbol{\alpha}}+\tilde{\beta}) \in A
$$


This implies property (ii). For the proof of property (iii) we define

$$
v(a)=P_{1}(u(a+\beta(a), \tau(a))-a),
$$

where $u\left(u_{0}, t\right)$ is the solution $(t \geqslant 0)$ of $(6.1)$ with $u\left(u_{0}, 0\right)=u_{0}$. This completes the proof of Theorem 6.3.

REMARKS. 1. The manifold $A$ is usually not invariant.

2. It is possible to obtain a result like Theorem 6.3 in the $C^{r}$ or $C^{\infty}$ category (see [31]). Due to the fact that the inverse of $\operatorname{grad} \Gamma$ is of the order $|a|^{2}$, there is some loss of differentiability of the period as a function of the radius $a$.

3. Introducing a near-identity transformation of the $b$-component prior to application of the implicit function theorem on the operator $\Gamma,(6.14)$ will yield a different analytic manifold which nevertheless has all of the properties listed in Theorem 6.3. The various analytic manifolds will all pass through the periodic solutions with period close to 1 . Therefore, if these solutions fill up the analytic manifold (i.e. $v \equiv 0$ on $A$ ) this manifold is unique, and invariant (see also Theorem 7.1).

4. Lemma 2 of [31] produces a smooth manifold and a finite-dimensional function $v$ such that the zeros of $v$ correspond to periodic solutions of (6.1) on the manifold. This manifold seems to be different from the one which we have constructed and from the other manifolds which have been mentioned in the previous remark.

Moser's procedure in [31] involves a Lyapunov-Schmidt type reduction as well as a coordinate change after which the equation for the periodic solution gets additional symmetry. For a related treatment of the nilpotent case $\left(L\left(B_{2}\right) \neq B_{2}\right)$, see [10].

5. Let $\operatorname{spec}\left(\left.L\right|_{B_{1}}\right)=\left\{ \pm 2 \pi \omega_{k} i: k=1, \ldots, m\right\}$. Then hypothesis $\mathrm{H}_{2}$ implies that the eigenvalues $\pm 2 \pi \omega_{k} i$ have equal algebraic and geometric multiplicities, and that $\omega_{k}$ is an integer. The manifold $A$ contains all normal mode solutions (with periods close to $\omega_{k}^{-1}$ ) as well as the resonant solutions between any subset of the frequencies provided these resonant solutions have a period close to $p^{-1}$, where $p$ is an integer.

6. If the operator $K_{n}=(\exp (n L)-1) i_{2}: B_{2} \rightarrow B_{2}$ also satisfies $\mathrm{H}_{3}$, then there exists an analytic manifold $A_{n}$ which contains all periodic solutions with period close to $n$, as well as a function $v_{n}: A_{n} \rightarrow B_{1}$ which is zero on $n$-periodic solutions. The manifolds $A_{n}$ and $A$ have in common the periodic solutions of period close to 1; otherwise $A_{n}$ and $A$ may be different. If $L$ possesses imaginary eigenvalues which are rationally independent from the eigenvalues of $\left.L\right|_{B_{1}}$, then $K_{n}^{-1}$ exists for all $n$, but $K_{n}^{-1}$ will occasionally have a large operator norm due to small divisors; therefore, the domain of definition of the manifolds $A_{n}$ may shrink to $0 \in B_{1}$ for $n \rightarrow \infty$. If there are eigenvalues of $L$ which rationally depend on those of $\left.L\right|_{B_{1}}$ but which are not in $\operatorname{spec}\left(\left.L\right|_{B_{1}}\right)$, then there is an $n$ such that $K_{n}^{-1}$ does not exist; one would have to enlarge the space $B_{1}$ with the critical eigenvectors in $B_{2}$ in order to treat solutions of periods $\geqslant n$.

7. There is an extensive literature (see e.g. $[\mathbf{1}, \mathbf{3 8}, \mathbf{5 0}]$ and the reference lists therein) on how to find periodic solutions near an equilibrium point. If we apply any one of the available techniques to the equation

$$
d x / d t=P_{1} L(x+\beta(x))+P_{1} N(x+\beta(x)) \quad\left(x \in B_{1}\right),
$$


and we thereby obtain a periodic solution with period close to $n$, then this solution will correspond to a zero of $v_{n}$ and hence to a periodic solution of (6.1).

For an analysis of Hamiltonian systems along this line which is directly based on the manifold and the function $v$ constructed in [31] and which studies the periodic solutions by using Birkhoff normal forms and unfolding of singularities, see [9].

7. The subcenter manifold. In this section we shall further investigate the analytic manifolds which have been constructed in $\$ 6$. In the case that these manifolds are two-dimensional and a nondegenerate integral exists, the manifolds turn out to be completely filled by periodic solutions: this is a new approach to Lyapunov's theorem (Theorem 7.1).

We conclude this section by giving some examples which illustrate the special properties of subcenter manifolds.

DEFINITION OF SUBCENTER MANIFOLD. Consider the system on the Banach space $B$

$$
d u / d t=L u+N(u) .
$$

We suppose that $L$ is linear in $B$ and $N$ is nonlinear of at least quadratic order. We also suppose that $B=B_{1} \oplus B_{2}, P_{i}(B)=B_{i}$ with $P_{1}+P_{2}=1, L\left(B_{i}\right) \subset B_{i}(i=1,2)$. Moreover, $\operatorname{Re}\left(\operatorname{spec}\left(\left.L\right|_{B_{1}}\right)\right)=0$ but we do not assume that the spectrum of $\left.L\right|_{B_{2}}$ stays away from the imaginary axis. An invariant manifold of (6.1) with the same dimension as $B_{1}$ which is tangent to $B_{1}$ in the origin is called a subcenter manifold (cf. [26]).

REMARK. If the spectrum of $\left.L\right|_{B_{2}}$ does stay away from the origin, then the manifold is of course a center manifold.

THEOREM 7.1. Consider system (7.1) and let $L$ and $B$ satisfy the hypotheses $\mathrm{H}_{1}$ and $\mathrm{H}_{3}$ of §6. Moreover, let $\operatorname{dim} B_{1}=2$ and

$$
\left.L\right|_{B_{1}}=2 \pi\left(\begin{array}{cc}
0 & 1 \\
-1 & 0
\end{array}\right) \text {. }
$$

Let $I$ be a $C^{2}$ function from $B$ to $\mathbf{R}$ such that for every solution of (7.1) we have $d I(u(t)) / d t=0$. Moreover, assume that $I(0)=0$ and $D I(0)=0$, where $D I$ is the Frechet derivative of $I$. Suppose that the bilinear form $I_{2}: B_{1} \times B_{1} \rightarrow \mathbf{R}$ defined by $I_{2}[p, q]=\left.D^{2} I\right|_{u=0}[p, q]$ is positive definite.

Then the two-dimensional manifold $A$ which has been described in Theorem 6.3 is invariant, unique and filled with an analytic one-parameter family of periodic solutions.

Proof. Let $V$ be defined as in the proof of Theorem 6.3, and let $a_{0} \in V$ be arbitrary. Let $e_{1}=P_{1} a_{0}$ and $e_{2}=P_{1} L_{1} a_{0}$. Then for every small $r \in \mathbf{R}$,

$$
u\left(r a_{0}+\beta\left(r a_{0}\right), \tau\left(r a_{0}\right)\right)-\left(r a_{0}+\beta\left(r a_{0}\right)\right)=\alpha(r) e_{1}+\gamma(r) e_{2}
$$

with $\alpha(r), \gamma(r), \beta\left(r a_{0}\right)$ real functions which are all of the order $r^{2}$. Equation (6.16) implies

$$
\left(P_{1}\left(\alpha(r) e_{1}+\gamma(r) e_{2}\right), L_{1} a_{0}\right)=0
$$

hence

$$
\gamma(r) \equiv 0
$$


We then have

$$
\begin{aligned}
0 & =I\left(u\left(r a_{0}+\beta\left(r a_{0}\right), \tau\left(r a_{0}\right)\right)\right)-I\left(r a_{0}+\beta\left(r a_{0}\right)\right) \\
& =\left.D_{1} I\right|_{r a_{0}+\beta\left(r a_{0}\right)}\left[\alpha(r) e_{1}\right]+O(\alpha(r))^{2} \\
& =I_{2}\left[\alpha(r) e_{1} ; P_{1} r a_{0}\right]+o(r \alpha(r))+O(\alpha(r))^{2}
\end{aligned}
$$

So,

$$
0=r \alpha(r) I_{2}\left[e_{1}, e_{1}\right]+o(r \alpha(r))+O(\alpha(r))^{2},
$$

which contradicts the assumption on $I_{2}$ unless $\alpha(r) \equiv 0$ which means that $v\left(r a_{0}\right) \equiv 0$.

The analytic family of periodic solutions is

$$
\left\{u\left(r a_{0}+\beta\left(r a_{0}\right) ; t\right): 0 \leqslant r<r_{0}\right\}
$$

for $r_{0}$ a small positive number. This completes the proof of Theorem 7.1.

REMARK. Theorem 7.1 was proved in [29] by Lyapunov under the additional assumption that the integral $I(u)$ is analytic. For an alternative proof of this "Lyapunov center theorem" see [44]. Both proofs use majorated convergence of power series as opposed to the present proof which is based on the implicit function argument for the operator $\Gamma,(6.14)$.

In [7] the Lyapunov subcenter manifold is constructed outside the origin using a return map argument (comparable to the proof of Theorem 6.3); with the aid of polar coordinates one proves [26] that this manifold is $C^{1}$ through the origin. A $C^{r}$-proof may be given when a Birkhoff normal form transformation precedes this analysis.

The Lyapunov subcenter theorem is also implied by [31], where the analyticity of the manifold is brought about by the use of the Lyapunov-Schmidt method. The main result of that paper is the assertion that a nonresonance condition is not essential for the existence of periodic solutions, although one still needs such a condition in order to prove that these solutions fill up two-dimensional manifolds. For results on Hamiltonians see $[31,32,37]$. For another recent proof of Lyapunov's theorem see [42].

REMARK. J. Moser [31, p. 728] gives an example where $\operatorname{spec}\left(L_{1}\right)=\{ \pm i\}$ and $\operatorname{spec}\left(L_{2}\right)=\{ \pm j i\}$ (here $j$ is a positive integer) while all other conditions of Theorem 7.1 are fulfilled. Because of the resonant eigenvalues the hypothesis $\mathrm{H}_{2}$ is violated and there is no manifold of periodic solutions tangent to $B_{1}$. Of course, if $j>1$ there is a "fast frequency" manifold tangent to $B_{2}$. If $j=1$, then there may be no periodic solutions at all [32].

COROLlARY 7.2. Let (6.1) have a nondegenerate integral. Suppose that the imaginary points in the spectrum of $L$ are $\left\{ \pm 2 \pi i \nu_{1}^{-1}, \ldots, \pm 2 \pi i \nu_{p}^{-1}\right\}$ with the $\nu_{i}$ rationally independent. Let $A^{(k)}$ be the two-dimensional analytic invariant manifold from Theorem 7.1 which is associated with the eigenvalues $\pm 2 \pi i \nu_{k}^{-1}$. Let $u_{\lambda}$ be a family of periodic solutions of (6.1) as in Theorem 6.1. Let the periods $T_{\lambda}$ satisfy either one of the following two assumptions:

(i) $T_{\lambda}$ is bounded;

(ii) $T_{\lambda}$ is continuous for $\lambda \neq 0$ and $T_{\lambda}\left\|u_{\lambda}\right\| \mid \rightarrow 0$ for $\lambda \rightarrow 0$.

Then for $\lambda$ small enough, $u_{\lambda}$ will belong to one of the manifolds $A^{(k)}$. 
Proof. If $T_{\lambda}$ satisfies $i$, then from Lemma 6.1(i) we conclude that, for small $\lambda, T_{\lambda}$ will be close to one of the sets $\left\{n \nu_{k}, 1 \leqslant n \leqslant \nu_{k}^{-1}\left(\sup T_{\lambda}\right)\right\}$, hence $u_{\lambda}$ will belong to a manifold $A_{n}^{(k)}$ (where $1 \leqslant n \leqslant \nu_{k}^{-1} \sup T_{\lambda}$ ). However, the manifolds $A_{n}^{(k)}$ are all two-dimensional and contain the periodic manifold $A^{(k)}$, so $A_{n}^{(k)}=A^{(k)}$ for all $n$. So for $\lambda$ small enough, $u_{\lambda}$ will be a normal mode.

If $T_{\lambda}$ satisfies (ii), then from Lemma 6.1(ii) we see that $T_{\lambda}$ approaches a number $n \nu_{k}$, and the proof ends as in the previous case.

Most of the proofs of the properties of center manifolds in the $\$ \S 1-5$ relied heavily on the exponential contraction of the operators $\exp \left(L_{2} t\right)$ and $\exp \left(-L_{3} t\right)$ for $t \rightarrow \infty$. In the subcenter situation these operators do not have that property and an existence proof of the subcenter manifold (in the absence of an integral as in Theorem 7.1) would have to be built on the attraction properties of the nonlinear terms in (6.1). For an analysis along this line for diffeomorphisms see [19].

We conclude this section with some examples which illustrate the differences between center manifolds and subcenter manifolds.

EXAmple 1. Consider the system

$$
\dot{x}=y\left(x^{2}+y^{2}\right), \quad \dot{y}=-x\left(x^{2}+y^{2}\right) .
$$

The trajectories of this system in the $(x, y)$-plane are circles around the origin. Therefore, no matter which choice of one-dimensional linear subspaces $B_{1}, B_{2}$ of $B=\mathbf{R}^{2}$ is made, there will be no subcenter manifold.

EXAMPLE 2. We consider a system in cylindrical coordinates:

$$
\dot{r}=-2 r z, \quad \dot{\phi}=-1, \quad \dot{z}=r^{2}-z^{2} .
$$

There is no two-dimensional subcenter manifold which is tangent to the plane $z=0$. Also, the behaviour close to the line $r=0$ is different from the situation for center manifolds.

Another example of a vector field without subcenter manifolds can be found in $[47, \S \mathrm{V}]$, where it was constructed for a different purpose. This example (in $\mathbf{R}^{5}$ ) has one zero eigenvalue and two rationally independent imaginary pairs, which lead to invariant surfaces like the ones in Example 2.

The following example which has no eigenvalues zero is essentially more complicated than the previous ones.

EXAmple 3. Consider the system

$$
\begin{gathered}
d x_{1} / d t=x_{2}+x_{1} g_{1}\left(x_{1}^{2}+x_{2}^{2}\right), \quad d x_{2} / d t=-x_{1}+x_{2} g_{1}\left(x_{1}^{2}+x_{2}^{2}\right), \\
d y_{1} / d t=\frac{1}{2} y_{2}, \quad d y_{2} / d t=-\frac{1}{2} y_{1}+g_{2}\left(x_{1}^{2}+x_{2}^{2}\right) .
\end{gathered}
$$

If $g_{1}$ and $g_{2}$ are such that (7.11) admits a nondegenerate integral, then Theorem 7.1 guarantees a subcenter manifold which is tangent to the $\left(x_{1}, x_{2}\right)$-plane.

However, we shall now construct $g_{1}$ and $g_{2}$ such that no invariant manifold exists which is tangent to this plane.

For $g_{1}: \mathbf{R} \rightarrow \mathbf{R}$ we take

$$
g_{1}(z)=\alpha z^{2} \exp \left(-\beta / z^{2}\right)
$$


where $\alpha, \beta$ will be determined in the sequel. Denote by $r(t ; a)$ the solution on $-\infty<t \leqslant 0$ of

$$
d r / d t=r g_{1}\left(r^{2}\right) ; \quad r(0 ; a)=a \in[0, \infty) \subset \mathbf{R} .
$$

Define $\xi(a)=r(-2 \pi, a)$; then $\xi$ is a smooth monotonously increasing function, $\xi$ : $[0, \infty) \rightarrow[0, \infty)$ and $\xi(0)=0$. Choose $p_{0} \in(0, \infty)$ and define the sequence $p_{n}$ $(n \geqslant 0)$ by $p_{n+1}=\xi\left(p_{n}\right)$. Then $p_{n}$ decreases to 0 for $n \rightarrow \infty$. By choosing $\alpha, \beta$ and $K$ appropriately (e.g., $\alpha<1 / 32 \pi, \beta=4$ and $K>3+\exp \left(4 / p_{0}^{4}\right)$ ), one gets

$$
p_{n}>\sqrt{2 / \ln (n+K)} \quad(n \geqslant 0) \text {. }
$$

Now define a bounded function $\tilde{g}_{2}$ from $[0, \infty)$ to $\mathbf{R}$ which is $C^{\infty}$ on $(0, \infty)$, changes sign in the points $p_{n}$ and nowhere else, and which has the properties

$$
\left|\int_{0}^{-2 \pi} \sin \frac{\tau}{2} \tilde{g}_{2}\left(r\left(\tau, p_{n}\right)\right) d \tau\right|>1
$$

and

$$
e^{-1 / z} \tilde{g}_{2}(z) \in C^{\infty}([0, \infty) \rightarrow \mathbf{R}) .
$$

Then define $g_{2}(z)=\exp (-1 / z) \tilde{g}_{2}(z)$. We now prove that for this choice of $g_{1}, g_{2}$ there is no subcenter manifold which is tangent to the $\left(x_{1}, x_{2}\right)$-plane.

Suppose that such a subcenter manifold exists and is of the form

$$
\left\{x+\sigma(x) \mid x=\left(x_{1}, x_{2}\right) \text { in a neighborhood } U \text { of } 0 \text { in } \mathbf{R}^{2}\right\} \text {. }
$$

Here $\sigma(x)=\left(\sigma_{1}(x), \sigma_{2}(x)\right)$ should be a continuous mapping from $\mathbf{R}^{2}$ to $\mathbf{R}^{2}$. There is an $l \in \mathbf{N}$ such that $p_{l} \in U$. For all $n \geqslant l$ the $\left(y_{1}, y_{2}\right)$ component of the solution of (7.11) through the point $\left(\sigma_{1}\left(p_{n}, 0\right), \sigma_{2}\left(p_{n}, 0\right)\right)$ is

$$
\begin{aligned}
\left(\begin{array}{l}
y_{1}(t) \\
y_{2}(t)
\end{array}\right)= & \left(\begin{array}{cc}
\cos t / 2 & \sin t / 2 \\
-\sin t / 2 & \cos t / 2
\end{array}\right) \\
& \times\left\{\left(\begin{array}{l}
\sigma_{1}\left(p_{n}, 0\right) \\
\sigma_{2}\left(p_{n}, 0\right)
\end{array}\right)+\int_{0}^{t}\left(\begin{array}{cc}
\cos \tau / 2 & -\sin \tau / 2 \\
\sin \tau / 2 & \cos \tau / 2
\end{array}\right)\left(\begin{array}{c}
0 \\
g_{2}\left(r^{2}\left(\tau, p_{n}\right)\right)
\end{array}\right)\right\} d \tau .
\end{aligned}
$$

Hence,

$$
\sigma_{1}\left(p_{n+1}, 0\right)=y_{1}(-2 \pi)=-\sigma_{1}\left(p_{n}, 0\right)+\int_{0}^{-2 \pi} \sin \frac{\tau}{2} g_{2}\left(r^{2}\left(\tau, p_{n}\right)\right) d \tau
$$

If we define the sequence of numbers $k_{m}(m \geqslant 0)$ by

$$
k_{m}=\int_{0}^{-2 \pi} \sin \frac{\tau}{2} g_{2}\left(r^{2}\left(\tau, p_{m}\right)\right) d \tau
$$

then (7.19) leads to

$$
(-1)^{n} \sigma_{1}\left(p_{n}, 0\right)=(-1)^{l} \sigma_{1}\left(p_{l}, 0\right)-\sum_{m=l}^{n-1}(-1)^{m} k_{m} .
$$

Because $\tilde{g}_{2}$ changes sign in every $p_{n}$, the numbers $(-1)^{m} k_{m}$ do not change sign. Moreover, from the definition of $g_{2}$ and the inequalities (7.14) and (7.15) we obtain

$$
\left|k_{m}\right|>\exp \left(-\frac{1}{\left(p_{m+1}\right)^{2}}\right)>\frac{1}{\sqrt{m+1+K}} .
$$


Hence, the series in the right-hand side of (7.21) diverges, which contradicts the requirement that $\sigma_{1}\left(p_{n}, 0\right)$ approaches 0 for $n \rightarrow \infty$. This completes the proof of nonexistence of a subcenter manifold.

REMARK. The above example heavily rests on the infinite number of zeros of the function $g_{2}$. For an analytic system, such a situation cannot occur and it would therefore be interesting to have an analytic example of the nonexistence of a subcenter manifold, with the linear part of the dynamical system as in (7.11). The rational dependence of the eigenvalues in (7.11) is not essential.

EXAMPLE 4. We consider the system of differential equations which on bipolar coordinates $x_{1}=r \cos \phi, x_{2}=r \sin \phi, x_{3}=\rho \cos \psi$ and $x_{4}=\rho \sin \psi$ looks like

$$
\begin{aligned}
& d r / d t=-r\left(r^{4}-10 r^{2} \rho^{2}+5 \rho^{4}\right), \\
& d \rho / d t=-\rho\left(5 r^{4}-10 r^{2} \rho^{2}+\rho^{4}\right), \\
& d \phi / d t=-\omega_{1}, \quad d \psi / d t=-\omega_{2} .
\end{aligned}
$$

First, there is a collection of subcenter manifolds tangent to $\rho=0$; the difference between two of these manifolds is of the order $r^{5}$. Hence, unlike center manifolds, subcenter manifolds may differ by a polynomial. The situation near $r=0$ is similar.

Secondly, the flow in any subcenter manifold which is tangent to either $\rho=0$ or $r=0$ approaches the origin for $t \rightarrow \infty$, but the origin is an unstable point of the full four-dimensional system.

\section{Proofs.}

8.1. Proof of Lemma 4.1. Suppose that the solution of (4.14) exists on the interval $t^{*} \leqslant t \leqslant 0$ for some $t^{*}<0$. Then on this interval

$$
\begin{aligned}
\|x(t ; \xi)\| & \leqslant(1-c t)^{p}\|\xi\|+K \int_{t}^{0}(1-c(t-\tau))^{p}\|x(\tau ; \xi)\|^{2} d \tau \\
& \leqslant(1-c t)^{p}\|\xi\|+K(1-c t)^{p} \int_{t}^{0}\|x(\tau ; \xi)\|^{2} d \tau .
\end{aligned}
$$

The function $f(t, \xi)$ satisfies the differential equation

$$
d f(t, \xi) / d t=-p c f /(1-c t)-K(1-c t)^{p} f^{2} ; \quad f(0, \xi)=\|\xi\|,
$$

which is equivalent with the integral equation

$$
f(t, \xi)=(1-c t)^{p}\|\xi\|+K(1-c t)^{p} \int_{t}^{0}(f(\xi, \tau))^{2} d \tau
$$

Comparison of (8.15) and (8.17) yields the statement in (i). The proof of (ii) follows easily.

We finally prove (iii). Let $\xi \in D_{r}$ and let $t>0$, then $\xi=x(-t ; x(t ; \xi))$. There are two possibilities: either $-t \geqslant t_{0}(\|x(t ; \xi)\|, r)$ or $-t<t_{0}(\|x(t ; \xi)\|, r)$. In the first case, from (4.17)

$$
\|\xi\| \leqslant \frac{(1+c t)^{p}\|x(t ; \xi)\|}{1-(K /(2 p+1) c)\left\{(1+c t)^{2 p+1}-1\right\}\|x(t ; \xi)\|} .
$$


The denominator in this expression is positive. Solving (8.18) for $\|x(t ; \xi)\|$ yields the inequality (4.20). In the other situation $\left(-t<t_{0}\right)$ we obtain, from (4.17) and the definition of $t_{0}$,

$$
r=\frac{\left(1-c t_{0}\right)^{p}\|x(t ; \xi)\|}{1-(K /(2 p+1) c)\|x(t ; \xi)\|\left\{\left(1-c t_{0}\right)^{2 p+1}-1\right\}}
$$

So

(8.20) $\|x(t ; \xi)\|=\left(\frac{K}{(2 p+1) c}\left\{\left(1-c t_{0}\right)^{2 p+1}-1\right\}+\frac{\left(1-c t_{0}\right)^{p}}{r}\right)^{-1}$.

Now using $-t<t_{0}$ and $\|\xi\|<r$ leads again to (4.20). This completes the proof of the lemma.

8.2. Proof of Theorem 4.2. Suppose that $\sigma_{A}(x)$ is defined on a neighborhood $V \subset B_{1}$ of 0 . On $V \times B_{2}$ we perform the transformation of coordinates $x=u_{1}$; $w=u_{2}-\sigma_{A}\left(u_{1}\right)$ and we obtain, from (2.6),

$$
d x / d t=L_{1} x+h_{1}(x, w), \quad d w / d t=L_{2} w+h_{2}(x, w),
$$

where

$$
\begin{aligned}
h_{1}(x, w)= & n_{1}\left(x, w+\sigma_{A}(x)\right), \\
h_{2}(x, w)= & n_{2}\left(x, w+\sigma_{A}(x)\right)+L_{2} \sigma_{A}(x) \\
& -\left(d \sigma_{A} / d x\right)\left(L_{1} x+n_{1}\left(x, w+\sigma_{A}(x)\right)\right) .
\end{aligned}
$$

Let $U_{r}$, for small $r>0$, denote a neighborhood of radius $r$ of 0 ; suppose $U_{2 r} \subset V$. For all $x \in U_{r}$, define $\tilde{\sigma}(x)=\sigma_{B}(x)-\sigma_{A}(x)$. Let $g_{i}^{*}$ denote a function which coincides with $h_{i}$ on $U_{r}$ and which is 0 outside $2 r$. Suppose that the functions $g_{i}^{*}$ $(i=1,2)$ are chosen in the spirit of Theorem 3.1 and in such a way that the manifold

$$
\left\{(x, \tilde{\sigma}(x)): x \in U_{r}\right\}
$$

is a submanifold of the global (i.e. defined for all $x \in B_{1}$ ) center manifold of

$$
d x / d t=L_{1} x+g_{1}^{*}(x, w), \quad d w / d t=L_{2} w+g_{2}^{*}(x, w) .
$$

(i) The case $p=0$. Let the sequence $\sigma_{n}$ of functions from $B_{1}$ to $B_{2}$ be defined by $\sigma_{0}=0$ and

$$
\sigma_{n+1}(x)=\int_{-\infty}^{0} e^{-L_{2} \tau} g_{2}^{*}\left(x\left(\tau ; \xi, \sigma_{n}\right), \sigma_{n}\left(x\left(\tau, \xi, \sigma_{n}\right)\right)\right) d \tau,
$$

where $x\left(t, \xi, \sigma_{n}\right)$ satisfies $(t \leqslant 0)$ :

$$
d x / d t=L_{1} x+g_{1}^{*}\left(x, \sigma_{n}(x)\right) ; \quad x(0)=\xi .
$$

From (2.34), (2.35) we know that there are numbers $R, l>0$ such that, for all $\xi, \xi_{1}$, $\xi_{2} \in B_{1}$ and integer $n$,

$$
\left\|\sigma_{n}(\xi)\right\|<R, \quad\left\|\sigma_{n}\left(\xi_{1}\right)-\sigma_{n}\left(\xi_{2}\right)\right\|<l\left\|\xi_{1}-\xi_{2}\right\| .
$$

Define $M_{1}$ by

$$
M_{1}=\max \left\{\left\|g_{2}^{*}(x, w)\right\| \cdot\|w\|^{-1}:\|x\|<r \text { and }\|w\|<R\right\} .
$$


This maximum exists due to the transformation from $u_{2}$ to $w$. Suppose that $r$ is so small that

$$
8 C_{2} M_{1}<\delta
$$

Here $C_{2}$ is the constant which appears in the estimate (2.8a). Let $\eta<r$ be such that $\|\xi\|<\eta$ implies

$$
\left|t_{0}(\xi, r)\right|>D /\|\xi\|
$$

For every $\xi \in U \equiv U_{\eta}$ we write

$$
\sigma_{n+1}(\xi)=\int_{t_{0}(\xi, r)}^{0} e^{-L_{2} \tau} h_{2}\left(x(\tau ; \xi), \sigma_{n}(x(\tau ; \xi))\right) d \tau+\Sigma_{n}(\xi)
$$

where

$$
\Sigma_{n}(\xi)=\int_{-\infty}^{t_{0}(\xi, r)} e^{-L_{2} \tau} g_{2}^{*}\left(x(\tau ; \xi), \sigma_{n}(x(\tau ; \xi))\right) d \tau .
$$

So $\left\|\Sigma_{n}(\xi)\right\| \leqslant C_{2} e^{\delta t_{0}(\xi, r)} M_{2}$ and

$$
M_{2}=1+\max _{\|x\|<2 r:\|\eta\|<R}\left\|g_{2}^{*}(x, \eta)\right\|
$$

the constant $\delta$ has been defined in (2.8a). Let

$$
K=\max \left\{\left\|h_{1}(x, \sigma(x))\right\| \cdot\|x\|^{-2}:\|x\|<r \text { and }\|\sigma(x)\|<l\|x\|\right\} .
$$

Then for $t_{0}(\xi, r) \leqslant t \leqslant 0$ (now using $p=0$ ) we get from Lemma 4.1(i)

$$
\|x(t ; \xi)\| \leqslant\|\xi\| /(1+K\|\xi\| t) .
$$

We finally prove by induction that

$$
\sigma_{n}(\xi) \leqslant D_{1} \exp \left(-D_{2}\|\xi\|^{-1}\right)
$$

where

$$
D_{1}=2 C_{2} M_{2} \text { and } D_{2}=\min \{D \delta, \delta / 2 K\} \text {. }
$$

Clearly, (8.36) is in order for $n=0$. Suppose (8.36) is satisfied for $\sigma_{n}$, then

$$
\begin{aligned}
\left\|\sigma_{n+1}(\xi)\right\| & \leqslant \int_{t_{0}(\xi, r)}^{0} C_{2} e^{\delta \tau} M_{1}\left\|\sigma_{n}(x(\tau ; \xi))\right\| d \tau+C_{2} M_{2} e^{\delta t_{0}(\xi, r)} \\
& \leqslant M_{1} C_{2} \int_{t_{0}(\xi, r)}^{0} e^{\delta \tau} D_{1} \exp \left(-\frac{D_{2}}{\|x(\tau ; \xi)\|}\right) d \tau+C_{2} M_{2} e^{-D \delta /\|\xi\|} \\
& \leqslant\left(D_{1} M_{1} C_{2} \int_{t_{0}(\xi, r)}^{0} e^{\left(-D_{2} K+\delta\right) \tau} d \tau+C_{2} M_{2}\right) e^{-D_{2} /\|\xi\|} \\
& \leqslant\left(D_{1} \frac{2 M_{1} C_{2}}{-D_{2} K+\delta}+C_{2} M_{2}\right) e^{-D_{2} /\|\xi\|}
\end{aligned}
$$

From (8.29) and (8.37) we now obtain (8.36) for $\sigma_{n+1}$, and we know from the proof of Theorem 2.1 that for $n \rightarrow \infty$ the sequence $\sigma_{n}$ approaches the unique center manifold of (8.24), so the assertion (4.23) follows for $\sigma_{B}(\xi)-\sigma_{A}(\xi)$.

(ii) The case $p \geqslant 1$. For every $\varepsilon$ with $0<\varepsilon \leqslant r$ define $R$ and $M_{1}$ by

$$
\begin{aligned}
R & =\max \left\{\left\|\sigma_{B}(x)-\sigma_{A}(x)\right\|:\|x\| \leqslant 2 \varepsilon\right\}, \\
M_{1} & =\max \left\{\left\|g_{2}^{*}(x, w)\right\| \cdot\|w\|^{-1}:\|x\| \leqslant 2 \varepsilon \text { and }\|w\| \leqslant R\right\} .
\end{aligned}
$$


Suppose that $\varepsilon$ is so small that

$$
C_{2} M_{1}<\delta / 2
$$

where $C_{2}$ is the constant which apprears in estimate $(2.8 \mathrm{a})$.

Now let $x_{0} \in U_{\varepsilon}$ and $w_{0}=\tilde{\sigma}\left(x_{0}\right)$; this implies that the solution $\left(x\left(t ;\left(x_{0}, w_{0}\right)\right)\right.$, $\left.w\left(t ;\left(x_{0}, w_{0}\right)\right)\right)$ of $(8.24)$ which passes through $\left(x_{0}, w_{0}\right)$ for $t=0$ is defined for all $t$, $-\infty<t<\infty$.

We first turn our attention to $x\left(t ;\left(x_{0}, w_{0}\right)\right)$. There are two possibilities:

Case 1. There is a $t_{1}<0$ such that $\left\|x\left(t_{1} ;\left(x_{0}, w_{0}\right)\right)\right\|=\varepsilon$ and $\left\|x\left(t ;\left(x_{0}, w_{0}\right)\right)\right\|<\varepsilon$ for all $t \in\left(t_{1}, 0\right]$. Denote by $K$ the number

$$
K=\max \left\{\left\|h_{1}(x, \sigma(x))\right\| \cdot\|x\|^{-2}:\|x\|<\varepsilon \text { and }\|\sigma(x)\|<l\|x\|\right\} .
$$

By Lemma 4.1(iii) we conclude that

$$
\begin{aligned}
& \left\|x\left(-t_{1} ;\left(x\left(t_{1} ;\left(x_{0}, w_{0}\right)\right), w\left(t_{1} ;\left(x_{0}, w_{0}\right)\right)\right)\right)\right\| \\
& \quad \geqslant\left(\frac{K}{(2 p+1) c}\left\{\left(1-c t_{1}\right)^{2 p+1}-1\right\}+\frac{\left(1-c t_{1}\right)^{p}}{\left\|x\left(t_{1} ;\left(x_{0}, w_{0}\right)\right)\right\|}\right)^{-1}
\end{aligned}
$$

This implies that

$$
\left\|x_{0}\right\| \geqslant b /\left(1-c t_{1}\right)^{2 p+1}
$$

where

$$
b=\min _{0 \leqslant t<\infty} \frac{(1+c t)^{2 p+1}}{(K /(2 p+1) c)\left\{(1+c t)^{2 p+1}-1\right\}+(1+c t)^{p} / \varepsilon} .
$$

We now study the $w$ component. Define the function $v(t)$ for $0 \leqslant t \leqslant t_{1}$ by $v(t)=w\left(t+t_{1} ;\left(x_{0}, w_{0}\right)\right)$.

$$
\begin{aligned}
\|v(t)\| & \leqslant e^{L_{2} t}\left\|\tilde{\sigma}\left(x\left(t_{1} ;\left(x_{0}, w_{0}\right)\right)\right)\right\|+\int_{0}^{t}\left\|e^{L_{2}(t-\tau)}\right\| M_{1}\|v(\tau)\| d \tau \\
& \leqslant C_{2} \operatorname{Re}^{-\delta t}+C_{2} M_{1} \int_{0}^{t} e^{-\delta(t-\tau)}\|v(\tau)\| d \tau .
\end{aligned}
$$

With Gronwall's lemma and (8.40),

$$
v(t) \leqslant C_{2} R \exp \left(C_{2} M_{1}-\delta\right) t<C_{2} R \exp (-\delta t / 2) .
$$

So

$$
\left\|w_{0}\right\| \leqslant C_{2} R \exp \left(\delta t_{1} / 2\right) .
$$

From (8.42) and (8.46) we conclude

(8.47) $\left\|\sigma_{B}\left(x_{0}\right)-\sigma_{A}\left(x_{0}\right)\right\|=\left\|w_{0}\right\| \leqslant C_{2} R \exp (\delta / 2 c) \exp \left(-\delta(b /\|x\|)^{1 /(2 p+1)} / 2 c\right)$; so if we put

$$
D_{1}=C_{2} R \exp (\delta / 2 c)
$$

and

$$
D_{2}=-\delta b^{1 /(2 p+1)} / 2 c
$$

then (4.24) is proved. 
Case 2. For all $t<0$ we have $\left\|x\left(t ;\left(x_{0}, w_{0}\right)\right)\right\|<\varepsilon$. As in (8.46) we obtain

$$
\left\|w_{0}\right\| \leqslant C_{2} R e^{\delta t / 2}
$$

for all $t<0$; this implies $\sigma_{A}\left(x_{0}\right)=\sigma_{B}\left(x_{0}\right)$; so Theorem 4.2 is proved.

REMARK. If $\left(x_{0}, w_{0}\right)$ is an arbitrary point in the set $\|x\| \leqslant 2 \varepsilon,\left\|w_{0}\right\| \leqslant R$ with the property that the trajectory of (8.21) through this point stays within this set for all $t \leqslant 0$, then the estimate (8.50) is valid too, implying $w_{0}=0$. Hence, every center manifold contains all solutions which are sufficiently small for $-\infty<t \leqslant 0$ (see also [11, Theorem 9.1]).

8.3. Proof of Corollary 4.3. We give the proof for $k=0$ only; the proof for $k \geqslant 1$ follows from the same argument. First of all, apply the transformation $x=u_{1}$, $w=u_{2}-\sigma_{A}\left(u_{1}\right)$ which changes (2.6) into

$$
d x / d t=L_{1} x+h_{1}(x, w), \quad d w / d t=L_{2} w+h_{2}(x, w),
$$

with $h_{i}(x, w)$ as in (8.22); we have $h_{2}(x, 0)=0$. Let $U_{r}$, for every $r>0$, denote a neighborhood of radius $r$ around the origin in $B_{1}$. Let $a>0$ and suppose $U_{2 a} \subset U$. From the proof of Theorem 3.1 we conclude that (8.51) can be modified outside $U_{a}$ in such a way that $\left\{(x, \tilde{\sigma}(x)): x \in B_{1}\right\}$ is the unique global center manifold of

$$
d x / d t=L_{1} x+g_{1}^{*}(x, w), \quad d w / d t=L_{2} w+g_{2}^{*}(x, w),
$$

where $\tilde{\sigma}(x)=\sigma_{B}(x)-\sigma_{A}(x)$, while $g_{i}^{*}$ coincides with $g_{i}$ on $U_{a} \times B_{2}$. Finally, Theorem 3.1 implies with $\tilde{\sigma}(x) \in C^{2}\left(\stackrel{\circ}{U} \rightarrow B_{2}\right)$ that the modified system still is $C^{1}$ outside the origin.

The function $\tilde{\sigma}(x)$ now satisfies

$$
(d \tilde{\sigma} / d x)\left[L_{1} x+g_{1}^{*}(x, \tilde{\sigma}(x))\right]=L_{2} \tilde{\sigma}(x)+g_{2}^{*}(x, \tilde{\sigma}(x)), \quad x \in B_{1} \backslash\{0\} .
$$

For $x \neq 0$ we can differentiate this expression with respect to $x$ :

$$
\begin{gathered}
\frac{d^{2} \tilde{\sigma}}{d x^{2}}\left[L_{1} x+g_{1}^{*}(x, \tilde{\sigma}(x)), \cdot\right]+\frac{d \tilde{\sigma}}{d x}\left[L_{1} \cdot+\frac{\partial g_{1}^{*}}{\partial x} \cdot+\frac{\partial g_{1}^{*}}{\partial w} \frac{d \tilde{\sigma}}{d x} \cdot\right] \\
=L_{2} \frac{d \tilde{\sigma}}{d x}[\cdot]+\frac{\partial g_{2}^{*}}{\partial x}[\cdot]+\frac{\partial g_{2}^{*}}{\partial w} \frac{d \tilde{\sigma}}{d x}[\cdot] .
\end{gathered}
$$

Now let the function $v(t)$ satisfy

$$
\frac{d v}{d t}=L_{1} v+\frac{\partial g_{1}^{*}}{\partial x}[v]+\frac{\partial g_{1}^{*}}{\partial w}[z]
$$

where

$$
z=(d \tilde{\sigma} / d x)[v] .
$$

Then from (8.54), (8.55) and (8.56) we obtain

$$
\frac{d z}{d t}=L_{2} z+\frac{\partial g_{2}^{*}}{\partial x}[v]+\frac{\partial g_{2}^{*}}{\partial w}[z]
$$

We consider the set of equations (8.51), (8.55), and (8.57). As in the proof of Theorem 4.2 we are able to obtain the inequalities for $t \leqslant 0$ :

$$
\|(z(t), w(t))\|_{B_{2} \times B_{2}} \leqslant c e^{\delta t / 2}
$$


and

$$
\|(x(t), v(t))\|_{B_{1} \times B_{1}} \geqslant b /(1-c t)^{2 p+1},
$$

from which we conclude that there are constants $D_{1}, \tilde{D}_{2}$ such that, for all $(x, v) \in$ $U_{a} \times U_{a}$,

$$
\|(d \tilde{\sigma} / d x)[v]\|=\|z\| \leqslant D_{1} \exp \left(-\tilde{D}_{2} /\|(x, v)\|^{1 /(2 p+1)}\right) .
$$

Now take $v=\|x\| f$ with $f \in B_{1},\|f\|=1$ but otherwise $f$ arbitrary. Then

$$
\begin{aligned}
\left\|\frac{d \tilde{\sigma}}{d x}[f]\right\| & \leqslant \frac{D_{1}}{\|x\|} \exp \left(-\frac{\tilde{D}_{2}}{\|x\|^{1 /(2 p+1)}\|(1, f)\|^{1 /(2 p+1)}}\right) \\
& \leqslant \frac{D_{1}}{\|x\|} \exp \left(-\frac{\tilde{D}_{2}}{2\|x\|^{1 /(2 p+1)}}\right)
\end{aligned}
$$

So

$$
\|d \tilde{\sigma} / d x\|_{\mathscr{L}\left(B_{1} \rightarrow B_{2}\right)} \leqslant D_{1} \exp \left(-D_{2} /\|x\|^{1 /(2 p+1)}\right),
$$

where $D_{2}$ such that for all $x,\|x\|<a$,

$$
-\tilde{D}_{2} / 2\|x\|^{1 /(2 p+1)}-\log \|x\| \leqslant-D_{2} /\|x\|^{1 /(2 p+1)} .
$$

(8.62) implies the continuity of $d \tilde{\sigma} / d x$ on $U$, which proves the corollary.

8.4. Proof of Theorem 6.1. We use the notation introduced in the proof of Theorem 6.2 and we start the proof of Theorem 6.1 from equation (6.9). Let $Q_{j}$ denote the projection of $B$ onto $\operatorname{ker}\left(L-2 \pi i \nu_{j}^{-1}\right)$. Then there is a constant $D$ such that, for small $\lambda$ and all $j, 1 \leqslant j \leqslant p$,

$$
\left|\exp \left(2 \pi i \nu_{j}^{-1} T_{\lambda}\right)-1\right|\left\|Q_{j} w_{\lambda}(0)\right\| \leqslant D \varepsilon_{\lambda} T_{\lambda} .
$$

Now let

$$
M=\sup \left(T_{\lambda}\||u|\|^{1 / l}\right)
$$

and

$$
V=\max \left\{\left|\nu_{j}\right|: 1 \leqslant j \leqslant p\right\} .
$$

Take a neighborhood of 0 in $\lambda$-space such that, for the corresponding $\varepsilon_{\lambda}$,

$$
\varepsilon_{\lambda}<(2 p)^{2 l /(k+1-l)}
$$

and also

$$
M^{-k} \frac{c V^{k}\left(\varepsilon_{\lambda}\right)^{k / l}}{2}-\frac{p V}{2 \pi} D M \varepsilon_{\lambda}^{1-1 / l}>\frac{V D M}{2 \pi}\left(\varepsilon_{\lambda}\right)^{(k+l-1) / 2 l} .
$$

This is possible because $k / l<(k+l-1) / 2 l$ and $k / l<1-1 / l$. Because $\left\|w_{\lambda}\right\|=1$ and $Q_{1}+\cdots+Q_{p}=1$ there is at least one $j_{0}$ (which may depend on $\lambda$ so we write $\left.j_{0}(\lambda)\right)$ such that

$$
\left\|Q_{j_{0}(\lambda)} w_{\lambda}(0)\right\| \geqslant 1 / p
$$

Hence, there also is an $n_{0}(\lambda)$ such that

$$
\left|\nu_{j_{0}(\lambda)}^{-1} T_{\lambda}-n_{0}(\lambda)\right| \leqslant \frac{p}{2 \pi} D \varepsilon_{\lambda} T_{\lambda} \leqslant \frac{p}{2 \pi} D M\left(\varepsilon_{\lambda}\right)^{1-1 / l}
$$


which implies that, for every pair $m, \nu_{j}$ with $m \neq n_{0}(\lambda)$ and $j \neq j_{0}(\lambda)$,

$$
\left|T_{\lambda}-m \nu_{j}\right|>\left|n_{0} \nu_{j_{0}}-m \nu_{j}\right|-(p V / 2 \pi) M D\left(\varepsilon_{\lambda}\right)^{1-1 / l} .
$$

Now, suppose that $n_{0}>m$ (the other case is treated similarly). Then

$$
\left|n_{0} \nu_{j_{0}}-m \nu_{j}\right|>\frac{c}{n_{0}^{k}}>\frac{c V^{k}}{2 T_{\lambda}^{k}}>\frac{c V^{k}}{2} \varepsilon_{\lambda}^{k / l} M^{-k}
$$

hence, with (8.68),

$$
\left|T_{\lambda}-m \nu_{j}\right|>\frac{V D}{2 \pi} M\left(\varepsilon_{\lambda}\right)^{(k+l-1) / 2 l}>\frac{V D}{2 \pi} T_{\lambda}\left(\varepsilon_{\lambda}\right)^{(k+l+1) / 2 l} .
$$

So

$$
\left\|Q_{j} w_{\lambda}(0)\right\|<\left(\varepsilon_{\lambda}\right)^{(l-k-1) / 2}<1 / 2 p .
$$

This implies that there is a gap of width at least $(2 p)^{-1}$ between $\left\|Q_{j_{0}(\lambda)^{w_{\lambda}}}(0)\right\|$ and the other components of $w_{\lambda}(0)$. From the continuity of $u_{\lambda}$ we conclude that there will be no jumps in $j_{0}(\lambda)$, which implies that $j_{0}$ does not depend on $\lambda$. Hence, for this single $j_{0}$ we have the estimate (8.70) for all $\lambda$ small enough, so the distance between $T_{\lambda}$ and the set $\left\{n \nu_{j_{0}}: n\right.$ an integer $\}$ is smaller than $(p V / 2 \pi) D \varepsilon_{\lambda}^{1-1 / l}$, which converges to 0 with $\lambda$. The actual multiple $n_{0}(\lambda)$ may not be constant because there may be period-doubling bifurcations in the family $u_{\lambda}$; this phenomenon is excluded if $T_{\lambda}$ happens to be continuous. This completes the proof of (iii).

Next, we prove (i). Let $S$ be a bound on $T_{\lambda}$ for $\lambda$ close to 0 . Define $C$ by

$$
C=\max \left\{\left|n \nu_{i}-m \nu_{j}\right|:\left|n \nu_{i}\right|<S+1 \text { and }\left|m \nu_{j}\right|<S+1\right\} \text {. }
$$

Obviously, there is a constant $M$ such that

$$
T_{\lambda}^{2} \varepsilon_{\lambda}<M \text {. }
$$

Now run the proof of (iii) again, with $k=0$ and $l=2$. For $\varepsilon_{\lambda}$ small enough, the same conclusions follow as before. This proves (i).

We finish by proving 6.1(ii). Define $N_{i}=\operatorname{entier}\left(\nu_{i}^{-1}\right)+1(1 \leqslant i \leqslant p)$ and

$$
N=N_{1}+\cdots+N_{p} \text {. }
$$

Now study in $\lambda$ space a neighborhood $\Lambda$ of 0 such that, for all $\lambda \in \Lambda(\lambda \neq 0)$,

$$
(D p V / 2 \pi) \varepsilon_{\lambda} T_{\lambda}<1 / 2 N \text {. }
$$

Choose $\lambda_{1}$ from this neighborhood. From (8.64) and (8.69) we conclude that there are integers $n_{0}\left(\lambda_{1}\right)$ and $j_{0}\left(\lambda_{1}\right)$ such that

$$
\left|T_{\lambda_{1}}-n_{0}\left(\lambda_{1}\right) \nu_{j_{0}\left(\lambda_{1}\right)}\right|<(p V D / 2 \pi) \varepsilon_{\lambda_{1}} T_{\lambda_{1}} \text {. }
$$

There is an interval of length $I$ in the half line $T \geqslant n_{0}\left(\lambda_{1}\right) \nu_{j_{0}\left(\lambda_{1}\right)}$ such that $I$ has length $N^{-1}$ and $I$ does not contain any point of the form $n \nu_{i}$, where $n$ is an integer and $1 \leqslant i \leqslant p$. Suppose that there is a $\lambda \in \Lambda$ for which $T_{\lambda}$ is the midpoint of $I$. Then again by (8.64) and (8.69) there must be a point $n \nu_{i}$ such that

$$
\left|T_{\lambda}-n \nu_{i}\right|<(p V / 2 \pi) D \varepsilon_{\lambda} T_{\lambda}<1 / 2 N
$$


This implies that $n \nu_{i} \in I$ which is a contradiction. Hence $T_{\lambda}$ cannot be the midpoint of $I$. By the continuity of $T_{\lambda}$ on the connected domain $\Lambda \backslash\{0\}$ we conclude that $T_{\lambda}$ is bounded on this domain. This completes the proof of 6.1(ii).

ACKNOWLEDGEMENT. The author wishes to express his gratitude for many stimulating discussions with Professors J. J. Duistermaat and W. Eckhaus.

\section{REFERENCES}

1. R. Abraham and J. E. Marsden, Foundations of mechanics, 2nd ed., Benjamin-Cummings, Reading, Mass., 1978.

2. V. I. Arnold, Loss of stability of self-oscillations close to resonance and versal deformations of equivariant vector fields, Functional Anal. Appl. 11 (1977), 85-92.

3. Y. N. Bibikov, Local theory of nonlinear analytic ordinary differential equations, Lecture Notes in Math., Vol. 702, Springer-Verlag, Berlin, 1979.

4. J. Carr, Applications of centre manifold theory, Lecture Notes, Brown University, Providence, R.I., 1979.

5. N. Chafee, $A$ bifurcation problem for a functional differential equation of finitely retarded type, J. Math. Anal. Appl. 35 (1971), 312-348.

6. E. Cotton, Sur les solutions asymptotiques des équations différentiels, Ann. Sci. Ecole Norm. Sup. 28 (1911), 473-521.

7. J. J. Duistermaat, On periodic solutions near equilibrium points of conservative systems, Arch. Rational Mech. Anal. 45 (1972), 143-160.

8. Stable manifolds, Preprint, University of Utrecht, Utrecht, The Netherlands, 1976.

9. Periodic solutions near equilibrium points of Hamiltonian systems, Preprint, University of Utrecht, Utrecht, The Netherlands, 1980.

10. J. J. Duistermaat and J. A. Vogel, The Moser-Weinstein method (work in progress).

11. N. Fenichel, Geometric singular perturbation theory for ordinary differential equations, J. Differential Equations 31 (1979), 53-98.

12. Center manifolds in bifurcation theory and singular perturbation theory, Preprint, University of British Columbia, Vancouver, B.C., Canada, 1978.

13. J. K. Hale, Ordinary differential equations, Wiley, New York, 1969.

14. P. Hartman, A lemma in the theory of structural stability of differential equations, Proc. Amer. Math. Soc. 11 (1960), 610-620.

15. B. Hassard, Bifurcation of periodic solutions of the Hodgkin-Huxley model for the Squid giant axon, J. Theoret. Biol. 71 (1978), 401-420.

16. B. Hassard and Y. H. Wan, Bifurcation formulae derived from center manifold theory, J. Math. Anal. Appl. 63 (1978), 297-312.

17. B. Hassard, N. D. Kazarinoff and Y. H. Wan, Theory and applications of Hopf bifurcation, esp. Appendix A, London Math. Soc. Lecture Notes, Vol. 41, Cambridge, 1981.

18. D. Henry, Geometric theory of semilinear parabolic equations, Lecture Notes, University of Kentucky,

19. M. W. Hirsch, C. C. Pugh and M. Shub, Invariant manifolds, Lecture Notes in Math., vol. 583, Springer-Verlag, Berlin, 1977.

20. P. J. Holmes and J. E. Marsden, Bifurcations to divergence and flutter in flow-induced oscillations: an infinite dimensional analy'sis, Automatica-J IFAC 14 (1978), 367-384.

21. P. J. Holmes, Editor, New approaches to nonlinear problems in dynamics, Proc. Asilomar Conf., 1979. SIAM, 1980.

22. (j. Iooss, Bifurcations of maps and applications, Math. Series 36, North-Holland, Amsterdam, 1979.

23. N. D. Kazarinoff, Y. H. Wan and P. van den Driessche, Hopf bifurcation and stability of periodic solutions of differential-difference and integro-differential equations, J. Inst. Math. Appl. 21 (1978), 461-478.

24. A. Kelley, The center manifold and integral manifolds for Hamiltonian systems, Notices Amer. Math. Soc. 12 (1965), 143-144.

25. The stable, center-stable, center, center-unstable, unstable manifolds, J. Differential Equations 3 (1967), 546-570.

26. On the Lyapunov sub-center manifold, J. Math. Anal. Appl. 18 (1967), 472-478

27. N. Kopell and L. N. Howard, Bifurcations and trajectories joining critical points, Adv. in Math. 18 (1975), 306-358. 
28. O. E. Lanford, III, Bifurcation of periodic solutions into invariant tori: the work of Ruelle and Takens, in Nonlinear Problems in the Physical Sciences and Biology (I. Stakgold, D. D. Joseph and D. H. Sattinger, eds.), Lecture Notes in Math., vol. 322, Springer-Verlag, Berlin, 1972, pp. 159-192.

29. A. Lyapunov, Problème général de la stabilité du mouvement, Ann. of Math. Studies, No. 17, Princeton Univ. Press, Princeton, N.J., 1949. (Russian original Kharkow, 1892.)

30. J. E. Marsden and M. McCracken, The Hopf bifurcation and its applications, Springer-Verlag, Berlin, 1976.

31. J. Moser, Periodic orbits near an equilibrium and a theorem by Alan Weinstein, Comm. Pure Appl. Math. 29 (1976), 727-747.

32. __ Addendum to [31], Comm. Pure Appl. Math. 31 (1978), 529-530.

33. P. Negrini and A. Tesei, Attractivity and Hopf bifurcation in Banach spaces, J. Math. Anal. Appl. 78 (1980), 204-221.

34. J. Palis and F. Takens, Topological equivalence of normally hyperbolic dynamical systems, Topology 16 (1977), 335-345.

35. O. Perron, Uher Stabilität und asymptotisches Verhalten der Integrale von Differentialgleichungssistemen, Math. Z. 29 (1928), 129-160.

36. V. A. Pliss, The reduction principle in the theory of stability of motion, Dokl. Akad. Nauk SSSR 154 (1964), 1044-1046; English transl., Soviet Math. 5 (1964), 247-250.

37. P. H. Rabinowitz, Periodic solutions of Hamiltonian systems, Comm. Pure Appl. Math. 31 (1978), $157-184$.

38. __ Periodic solutions of Hamiltonian systems: a survey, Preprint, University of Wisconsin, 1980.

39. R. D. Richtmyer, Invariant manifolds and bifurcations in the Tavlor problem (work in progress).

40. D. Ruelle and F. Takens, On the nature of turbulence, Comm. Math. Phys. 20 (1971), 167-192.

41. D. H. Sattinger, Topics in stability and bifurcation theory, Lecture Notes in Math., vol. 309. Springer-Verlag, Berlin, 1973.

42. D. S. Schmidt, Hopf's bifurcation theorem and the center theorem of Lyapunov with resonance cases, J. Math. Anal. Appl. 63 (1978), 354-370.

43. P. R. Sethna, Bifurcation theory and averaging in mechanical systems, in [21].

44. C. L. Siegel and J. K. Moser, Lectures on celestial mechanics, Springer-Verlag, Berlin, 1971.

45. J. Sijbrand, Studies in nonlinear stability and bifurcation theory, Chapter III, Ph.D. Thesis, 1981.

46. S. J. van Strien, Center manifolds are not $C^{\infty}$, Math. Z. 166 (1979), 143-145.

47. F. Takens, Singularities of vector fields, Inst. Hautes Études Sci. Publ. Math. 43 (1973), 47-100.

48. Applications of global analysis. I, Comm. Math. Inst. Utrecht 3 (1974).

49. M. M. Vainberg and V. A. Trenogin, Theory of branching of solutions of nonlinear equations, Noordhoff, Leiden, 1974. (Russian original Moscow, 1969.)

50. F. Verhulst, Discrete symmetric dynamical systems at the main resonances with applications to axi-symmetric galaxies, Philos. Trans. Roy. Soc. London Ser. A 290 (1979), 435-465.

51. Y. H. Wan, On the uniqueness of invariant manifolds, J. Differential Equations 24 (1977), 268-273.

Mathematisch InstituUt, RiJksuniversiteit Utrecht, Utrecht, THE Netherlands

Current address: MSE Department, Shell Laboratories, Badhuisweg 3, 1031 CM Amsterdam, The Netherlands 